\title{
PSEUDO-DIFFERENTIAL CALCULUS IN A BARGMANN SETTING
}

\author{
Nenad Teofanov and Joachim Toft \\ University of Novi Sad, Department of Mathematics and Informatics \\ Novi Sad, Serbia; nenad.teofanov@dmi.uns.ac.rs \\ Linnæus University, Department of Mathematics \\ Växjö, Sweden; joachim.toft@lnu.se
}

\begin{abstract}
We give a fundament for Berezin's analytic $\Psi$ do considered in [4] in terms of Bargmann images of Pilipović spaces. We deduce basic continuity results for such $\Psi$ do, especially when the operator kernels are in suitable mixed weighted Lebesgue spaces and act on certain weighted Lebesgue spaces of entire functions. In particular, we show how these results imply wellknown continuity results for real $\Psi$ do with symbols in modulation spaces, when acting on other modulation spaces.
\end{abstract}

\section{Introduction}

The aim of the paper is to put a fundament for the theory of analytic pseudodifferential operators, considered in [4] by Berezin. This is essentially done through a detailed analysis of Bargmann images of the so-called Pilipovic spaces of functions and distributions, given in $[11,26]$. More precisely, we consider kernels related to integral representations of analytic pseudo-differential operators to deduce their continuity properties. When the corresponding symbols belong to suitable (weighted) Lebesgue spaces of semi-conjugate analytic functions, we prove the continuity of the analytic pseudo-differential operators when acting between (weighted) Lebesgue spaces of analytic functions. Moreover, by using the relationship between the Bargmann transform and the short-time Fourier transform we show that our results can be used to recover well-known (sharp) continuity properties of (real) pseudo-differential operators with symbols in modulation spaces which act between other modulation spaces, see $[23,25,28]$. We emphasize that our approach here is more general, because we have relaxed the assumptions on the involved weight functions, compared to earlier contributions.

Analytic pseudo-differential operators, considered in [4] by Berezin are welldesigned when considering several problems in analysis and its applications, e.g. in quantum mechanics. In the context of abstract harmonic analysis it follows that any linear and continuous operator between Fourier invariant function and (ultra-) distribution spaces may, in a unique way, be transformed into an analytic pseudodifferential operator by the Bargmann transform (see Section 2). An advantage of such reformulations is that all of the involved objects are essentially entire functions and thereby possess several strong and convenient properties.

https://doi.org/10.5186/aasfm.2020.4512

2010 Mathematics Subject Classification: Primary 32W25, 35S05, 32A17, 46F05, 42B35; Secondary 32A25, 32A05.

Key words: Analytic kernels, Berezin operators, Pilipović spaces, modulation spaces, GelfandShilov spaces.

Acknowledgement: Nenad Teofanov was supported by Ministry of Education, Science and Technological Development of Serbia through the Project No 174024. 
The definition of analytic pseudo-differential operators resembles the definition of real pseudo-differential operators. In fact, let $a(x, \xi)$ be a suitable function or (ultra-)distribution on the phase space $\mathbf{R}^{2 d}$. Then the (real) pseudo-differential operator $\mathrm{Op}(a)$ acting on suitable sets of functions or (ultra-)distributions on the configuration space $\mathbf{R}^{d}$ is given by

$$
f(x) \mapsto(\mathrm{Op}(a) f)(x)=(2 \pi)^{-\frac{d}{2}} \int_{\mathbf{R}^{d}} a(x, \xi) \widehat{f}(\xi) e^{i\langle x, \xi\rangle} d \xi
$$

Here the integral in (0.1) should be interpreted in a distributional (weak) sense, if necessary, and we refer to [15] or Section 1 for the notation.

Suppose instead that $a$ is a suitable semi-conjugate entire (analytic) function on $\mathbf{C}^{d} \times \mathbf{C}^{d}=\mathbf{C}^{2 d}$, i.e. $(z, w) \mapsto a(z, \bar{w})$ is an entire (analytic) function. Then the analytic pseudo-differential operator $\mathrm{Op}_{\mathfrak{V}}(a)$ acting on suitable entire functions $F$ on $\mathbf{C}^{d}$ is given by

$$
F(z) \mapsto\left(\mathrm{Op}_{\mathfrak{V}}(a) F\right)(z)=\int_{\mathbf{C}^{d}} a(z, w) F(w) e^{(z, w)} d \mu(w)
$$

Here $d \mu(w)$ is the Gauss measure $\pi^{-d} e^{-|w|^{2}} d \lambda(w)$, where $d \lambda(w)$ is the Lebesgue measure on $\mathbf{C}^{d}$, and $(z, w)=\sum_{j=1}^{d} z_{j} \cdot \overline{w_{j}}$, when $z=\left(z_{1}, \ldots, z_{d}\right) \in \mathbf{C}^{d}$, and $w=$ $\left(w_{1}, \ldots, w_{d}\right) \in \mathbf{C}^{d}$. This means that the operator kernel (with respect to $d \mu$ ) is given by

$$
K(z, w)=K_{a}(z, w)=a(z, w) e^{(z, w)} .
$$

Evidently, $\left(\mathrm{Op}_{\mathfrak{V}}(a) F\right)(z)$ is equal to the integral operator

$$
\left(T_{K} F\right)(z)=\int_{\mathbf{C}^{d}} K(z, w) F(w) d \mu(w)
$$

with respect to $d \mu$, when $K$ is given by (0.3). By the analyticity properties of the symbol $a$ it follows that $(z, w) \mapsto K(z, \bar{w})$ is an entire function on $\mathbf{C}^{2 d}$.

In $[4,25]$ several facts of analytic pseudo-differential operators are deduced. For example, if $a$ and $F$ are chosen such that

$$
z \mapsto a(z, \cdot) F \cdot e^{(z, \cdot)}
$$

is locally uniformly bounded and analytic from $\mathbf{C}^{d}$ to $L^{1}(d \mu)$, then $\mathrm{Op}_{\mathfrak{V}}(a) F$ in $(0.2)$ is a well-defined entire function on $\mathbf{C}^{d}$. In $[4,25]$ it is also observed that

$$
\left(\mathrm{Op}_{\mathfrak{V}}\left(z_{j}\right) F\right)(z)=z_{j} F(z) \text { and }\left(\mathrm{Op}_{\mathfrak{V}}\left(\bar{w}_{j}\right) F\right)(z)=\left(\partial_{j} F\right)(z)
$$

when $F \in L^{1}\left(d \mu_{1}\right) \cap A\left(\mathbf{C}^{d}\right)$, and $d \mu_{1}(w)=(1+|w|) d \mu(w)$.

In such setting we study the mapping properties for complex integral operators and pseudo-differential operators when respectively $K=K_{a}$ and $a$ above belong to suitable classes of semi-conjugate entire functions. In fact, we permit more generally that $K$ and $a$ belong to suitable classes of formal semi-conjugate analytic power series expansions. That is, $K(z, w)$ and $a(z, w)$ are of the forms

$$
\sum_{\alpha, \beta} c_{K}(\alpha, \beta) e_{\alpha}(z) e_{\beta}(\bar{w}) \quad \text { and } \quad \sum_{\alpha, \beta} c_{a}(\alpha, \beta) e_{\alpha}(z) e_{\beta}(\bar{w}), \quad e_{\alpha}(z)=\frac{z^{\alpha}}{\sqrt{\alpha !}},
$$

respectively.

To set the stage for our study we collect the background material in Section 1. It contains a brief account on weight functions, Gelfand-Shilov spaces, spaces of Hermite functions and power series expansions, modulation spaces, and Bargmann 
transform and spaces of analytic functions. Especially, we recall basic facts for the spaces

$$
\mathcal{A}_{b_{\sigma}}\left(\mathbf{C}^{d}\right)\left(\mathcal{A}_{0, b_{\sigma}}\left(\mathbf{C}^{d}\right)\right), \quad \mathcal{A}_{s}\left(\mathbf{C}^{d}\right)\left(\mathcal{A}_{0, s}\left(\mathbf{C}^{d}\right)\right),
$$

and their Bargmann duals

$$
\mathcal{A}_{b_{\sigma}}^{\prime}\left(\mathbf{C}^{d}\right)\left(\mathcal{A}_{0, b_{\sigma}}^{\prime}\left(\mathbf{C}^{d}\right)\right), \quad \mathcal{A}_{s}^{\prime}\left(\mathbf{C}^{d}\right)\left(\mathcal{A}_{0, s}^{\prime}\left(\mathbf{C}^{d}\right)\right),
$$

when $s, \sigma>0$. The spaces in (0.6) consist of all formal power series

$$
F(z)=\sum_{\alpha} c(F, \alpha) e_{\alpha}(z)
$$

with coefficients satisfying

$$
|c(F, \alpha)| \lesssim h^{|\alpha|} \alpha !^{-\frac{1}{2 \sigma}}, \quad|c(F, \alpha)| \lesssim e^{-r|\alpha| \frac{1}{2 s}},
$$

respectively, for some (for every) $h, r>0$, and the spaces in (0.7) consist of all formal power series in $(0.8)$ such that

$$
|c(F, \alpha)| \lesssim h^{|\alpha|} \alpha !^{+\frac{1}{2 \sigma}}, \quad|c(F, \alpha)| \lesssim e^{+r|\alpha|^{\frac{1}{2 s}}}
$$

respectively, for every (for some) $h, r>0$.

In Section 2 we extend the definition of (0.4) to allow the kernels $K$ to belong to any of the spaces

$$
\hat{\mathcal{A}}_{0, b_{\sigma}}\left(\mathbf{C}^{2 d}\right), \quad \hat{\mathcal{A}}_{b_{\sigma}}\left(\mathbf{C}^{2 d}\right), \quad \hat{\mathcal{A}}_{0, s}\left(\mathbf{C}^{2 d}\right), \quad \hat{\mathcal{A}}_{s}\left(\mathbf{C}^{2 d}\right)
$$

and their duals

$$
\widehat{\mathcal{A}}_{0, b_{\sigma}}^{\prime}\left(\mathbf{C}^{2 d}\right), \quad \widehat{\mathcal{A}}_{b_{\sigma}}^{\prime}\left(\mathbf{C}^{2 d}\right), \quad \widehat{\mathcal{A}}_{0, s}^{\prime}\left(\mathbf{C}^{2 d}\right), \quad \hat{\mathcal{A}}_{s}^{\prime}\left(\mathbf{C}^{2 d}\right),
$$

where

$$
\widehat{\mathcal{A}}_{b_{\sigma}}\left(\mathbf{C}^{2 d}\right)=\left\{K ;(z, w) \mapsto K(z, \bar{w}) \in \mathcal{A}_{b_{\sigma}}\left(\mathbf{C}^{2 d}\right)\right\},
$$

and similarly for the other spaces in (0.9) and (0.10). In the end we prove that if $s>0$ or $s=b_{\sigma}$, then the integral operators in $(0.4)$,

$$
T_{K}: \mathcal{A}_{s}\left(\mathbf{C}^{d}\right) \mapsto \mathcal{A}_{s}^{\prime}\left(\mathbf{C}^{d}\right) \quad \text { when } \quad K \in \widehat{\mathcal{A}}_{s}^{\prime}\left(\mathbf{C}^{2 d}\right),
$$

and

$$
T_{K}: \mathcal{A}_{0, s}\left(\mathbf{C}^{d}\right) \mapsto \mathcal{A}_{0, s}^{\prime}\left(\mathbf{C}^{d}\right) \quad \text { when } \quad K \in \widehat{\mathcal{A}}_{0, s}^{\prime}\left(\mathbf{C}^{2 d}\right),
$$

are uniquely defined and continuous, and similarly when the roles of the non-duals in (0.6) and (0.9), and their duals in (0.7) and (0.10) are swapped. We also prove the opposite direction, that any linear and continuous operators between such spaces are given by such kernel operators. These kernel results are given in Propositions 2.2 and 2.3. Due to the Bargmann transform homeomorphisms, these results are also equivalent to Theorems 3.3 and 3.4 in [7] on kernel theorems for Pilipović spaces. (See Subsection 1.5.)

Note that, if $s \geqslant \frac{1}{2}$, then the spaces of power series expansions above can be identified with certain spaces of analytic and semi-conjugate analytic functions. For 
example we have

$$
\begin{aligned}
& \mathcal{A}_{b_{\sigma}}\left(\mathbf{C}^{d}\right)=\left\{F \in A\left(\mathbf{C}^{d}\right) ;|F(z)| \lesssim e^{r|z|^{\frac{2 \sigma}{\sigma+1}}} \text { for some } r>0\right\}, \quad \sigma>0, \\
& \mathcal{A}_{s}\left(\mathbf{C}^{d}\right)=\left\{F \in A\left(\mathbf{C}^{d}\right) ;|F(z)| \lesssim e^{\frac{1}{2} \cdot|z|^{2}-r|z|^{\frac{1}{2 s}}} \text { for some } r>0\right\} \\
& \mathcal{A}_{s}^{\prime}\left(\mathbf{C}^{d}\right)=\left\{F \in A\left(\mathbf{C}^{d}\right) ;|F(z)| \lesssim e^{\frac{1}{2} \cdot|z|^{2}+r|z| \frac{1}{2 s}} \text { for every } r>0\right\} \\
& \mathcal{A}_{b_{\sigma}}^{\prime}\left(\mathbf{C}^{d}\right)=\left\{F \in A\left(\mathbf{C}^{d}\right) ;|F(z)| \lesssim e^{r|z|^{\frac{2 \sigma}{\sigma-1}}} \text { for every } r>0\right\}, \quad \sigma>1, \\
& \mathcal{A}_{b_{1}}^{\prime}\left(\mathbf{C}^{d}\right)=A\left(\mathbf{C}^{d}\right) \text { and } \mathcal{A}_{0, b_{1}}^{\prime}\left(\mathbf{C}^{d}\right)=A_{d}(\{0\}),
\end{aligned}
$$

and similarly for $\hat{\mathcal{A}}_{s}\left(\mathbf{C}^{2 d}\right)$ and $\hat{\mathcal{A}}_{s}^{\prime}\left(\mathbf{C}^{2 d}\right)$. In particular, the mappings (0.11) and (0.12) can be formulated in terms of those function spaces.

If instead $s \in\left(0, \frac{1}{2}\right]$ and $\sigma>0$, and $t \in \mathbf{C}$, then $K(z, w) \mapsto K(z, w) e^{t(z, w)}$ is homeomorphic on

$$
\widehat{A}_{b_{\sigma}}^{\prime}\left(\mathbf{C}^{2 d}\right), \quad \widehat{A}_{0, b_{\sigma}}^{\prime}\left(\mathbf{C}^{2 d}\right), \quad \widehat{A}_{s}^{\prime}\left(\mathbf{C}^{2 d}\right) \quad \text { and } \quad \widehat{A}_{0, s}^{\prime}\left(\mathbf{C}^{2 d}\right),
$$

see Theorem 2.6. In particular, (0.3) implies that the mappings (0.11) and (0.12) still hold true with $\mathrm{Op}_{\mathfrak{V}}(a)$ in place of $T_{K}$. (Cf. Theorems 2.7 and 2.8.)

In the case $s \geqslant \frac{1}{2}$, the conditions on $a$ and its kernel $K_{a}$ of $\operatorname{Op}_{\mathfrak{V}}(a)$ are slightly different. More precisely, these conditions are of the form

$$
|a(z, w)| \lesssim e^{\frac{1}{2} \cdot|z-w|^{2}+r\left(|z|^{\frac{1}{2 s}}+|w|^{\frac{1}{2 s}}\right)}
$$

and

$$
|K(z, w)| \lesssim e^{\frac{1}{2} \cdot\left(|z|^{2}+|w|^{2}\right)+r\left(|z|^{\frac{1}{2 s}}+|w|^{\frac{1}{2 s}}\right)}
$$

in order for the mappings (0.11) and (0.12) should hold. (Cf. Theorems 2.9 and 2.10.)

In Section 3 we consider operators (0.4), where certain linear pullbacks of their kernels obey suitable mixed and weighted Lebesgue norm estimates. We prove that such operators are continuous between appropriate (weighted) Lebesgue spaces of entire functions. For example, let $\omega$ be a weight on $\mathbf{C}^{d} \times \mathbf{C}^{d}$ and $\omega_{1}, \omega_{2}$ be weights on $\mathbf{C}^{d}$ such that

$$
\frac{\omega_{2}(z)}{\omega_{1}(w)} \lesssim \omega(z, w)
$$

and let

$$
G_{K, \omega}(z, w)=K_{\omega}(z, z+w)
$$

where

$$
K_{\omega}(z, w)=e^{-\frac{1}{2}\left(|z|^{2}+|w|^{2}\right)}|K(z, w)| \omega(\sqrt{2} \bar{z}, \sqrt{2} w) .
$$

If $p, q, p_{j}, q_{j} \in[1, \infty]$ satisfy

$$
\frac{1}{p_{1}}-\frac{1}{p_{2}}=\frac{1}{q_{1}}-\frac{1}{q_{2}}=1-\frac{1}{p}-\frac{1}{q} \text { and } q \leqslant p,
$$

and $G_{K, \omega} \in L^{p, q}\left(\mathbf{C}^{d} \times \mathbf{C}^{d}\right)$, then it follows from Theorem 3.3 that $T_{K}$ is continuous from $A_{E,\left(\omega_{1}\right)}^{\boldsymbol{p}_{1}}\left(\mathbf{C}^{d}\right)$ to $A_{E,\left(\omega_{2}\right)}^{\boldsymbol{p}_{2}}\left(\mathbf{C}^{d}\right)$. By slightly modifying the definition of $G_{K, \omega}$ we also deduce another similar but different continuity result where the condition $q \leqslant p$ above is removed (cf. Theorem 3.5).

We also present some consequences of these results. Theorem 3.4 can be considered as a special case of Theorem 3.3 formulated by analytic pseudo-differential 
operators instead of integral operators. Theorems 3.8 and 3.9 are obtained by imposing conditions on moderateness on $\omega, \omega_{1}$ and $\omega_{2}$ above and translating Theorem 3.3 and 3.5 to real pseudo-differential operators via the Bargmann transform and its inverse. These approaches show that obtained continuity results on analytic pseudo-differential or integral operators might be suitable when investigating real pseudo-differential operators. In fact, Theorems 3.8 and 3.9 agree with the sharp results [24, Theorem 3.3], [27, Theorem 3.1] and [28, Theorem 2.2] in the Banach space case. Remark 3.10 in the end of Section 3 shows that our approach can be used to extend the latter results on real pseudo-differential operators to include situations with non-moderate weights. We note that the moderate condition on weights may in some situations be significantly restrictive (cf. Remark 1.14 in Section 1).

\section{Preliminaries}

In this section we recall some facts on involved function and distribution spaces as well as on pseudo-differential operators. In Subsection 1.1 we introduce suitable weight classes. Thereafter we recall in Subsections 1.2-1.4 the definitions and basic properties for Gelfand-Shilov, Pilipović and modulation spaces. Then we discuss in Subsection 1.5 the Bargmann transform and recall some topological spaces of entire functions or power series expansions on $\mathbf{C}^{d}$. The section is concluded with a review of some facts on pseudo-differential operators.

1.1. Weight functions. A weight on $\mathbf{R}^{d}$ is a positive function $\omega \in L_{\text {loc }}^{\infty}\left(\mathbf{R}^{d}\right)$ such that $1 / \omega \in L_{\text {loc }}^{\infty}\left(\mathbf{R}^{d}\right)$. The weight $\omega$ on $\mathbf{R}^{d}$ is called moderate if there is a positive locally bounded function $v$ on $\mathbf{R}^{d}$ such that

$$
\omega(x+y) \leqslant C \omega(x) v(y), \quad x, y \in \mathbf{R}^{d},
$$

for some constant $C \geqslant 1$. If $\omega$ and $v$ are weights on $\mathbf{R}^{d}$ such that (1.1) holds, then $\omega$ is also called $v$-moderate. The set of all moderate weights on $\mathbf{R}^{d}$ is denoted by $\mathscr{P}_{E}\left(\mathbf{R}^{d}\right)$.

The weight $v$ on $\mathbf{R}^{d}$ is called submultiplicative, if it is even and (1.1) holds for $\omega=v$. From now on, $v$ always denotes a submultiplicative weight if nothing else is stated. In particular, if (1.1) holds and $v$ is submultiplicative, then it follows by straight-forward computations that

$$
\begin{aligned}
\frac{\omega(x)}{v(y)} & \lesssim \omega(x+y) \lesssim \omega(x) v(y), \\
v(x+y) & \lesssim v(x) v(y) \text { and } v(x)=v(-x), \quad x, y \in \mathbf{R}^{d} .
\end{aligned}
$$

Here and in what follows we write $A(\theta) \lesssim B(\theta), \theta \in \Omega$, if there is a constant $c>0$ such that $A(\theta) \leqslant c B(\theta)$ for all $\theta \in \Omega$.

If $\omega$ is a moderate weight on $\mathbf{R}^{d}$, then by [25] and above, there is a submultiplicative weight $v$ on $\mathbf{R}^{d}$ such that (1.1) and (1.2) hold (see also [13, 25]). Moreover if $v$ is submultiplicative on $\mathbf{R}^{d}$, then

$$
1 \lesssim v(x) \lesssim e^{r|x|}
$$

for some constant $r>0$ (cf. [13]). In particular, if $\omega$ is moderate, then

$$
\omega(x+y) \lesssim \omega(x) e^{r|y|} \text { and } e^{-r|x|} \leqslant \omega(x) \lesssim e^{r|x|}, \quad x, y \in \mathbf{R}^{d}
$$

for some $r>0$. 
1.2. Gelfand-Shilov spaces. Let $0<s \in \mathbf{R}$ be fixed. Then the (Fourier invariant) Gelfand-Shilov space $\mathcal{S}_{s}\left(\mathbf{R}^{d}\right)\left(\Sigma_{s}\left(\mathbf{R}^{d}\right)\right)$ of Roumieu type (Beurling type) consists of all $f \in C^{\infty}\left(\mathbf{R}^{d}\right)$ such that

$$
\|f\|_{\mathcal{S}_{s, h}} \equiv \sup \frac{\left|x^{\alpha} \partial^{\beta} f(x)\right|}{h^{|\alpha+\beta|}(\alpha ! \beta !)^{s}}
$$

is finite for some $h>0$ (for every $h>0$ ). Here the supremum should be taken over all $\alpha, \beta \in \mathbf{N}^{d}$ and $x \in \mathbf{R}^{d}$. The semi-norms $\|\cdot\|_{\mathcal{S}_{s, h}}$ induce an inductive limit topology for the space $\mathcal{S}_{s}\left(\mathbf{R}^{d}\right)$ and projective limit topology for $\Sigma_{s}\left(\mathbf{R}^{d}\right)$, and the latter space becomes a Fréchet space under this topology.

The space $\mathcal{S}_{s}\left(\mathbf{R}^{d}\right) \neq\{0\}\left(\Sigma_{s}\left(\mathbf{R}^{d}\right) \neq\{0\}\right)$, if and only if $s \geqslant \frac{1}{2}\left(s>\frac{1}{2}\right)$. The Gelfand-Shilov distribution spaces $\mathcal{S}_{s}^{\prime}\left(\mathbf{R}^{d}\right)$ and $\Sigma_{s}^{\prime}\left(\mathbf{R}^{d}\right)$ are the dual spaces of $\mathcal{S}_{s}\left(\mathbf{R}^{d}\right)$ and $\Sigma_{s}\left(\mathbf{R}^{d}\right)$, respectively. We have

$$
\begin{aligned}
\mathcal{S}_{1 / 2}\left(\mathbf{R}^{d}\right) & \hookrightarrow \Sigma_{s}\left(\mathbf{R}^{d}\right) \\
& \left.\hookrightarrow \mathcal{S}_{s}\left(\mathbf{R}^{d}\right) \hookrightarrow \mathscr{R}^{d}\right) \hookrightarrow \Sigma_{t}\left(\mathbf{R}^{d}\right) \hookrightarrow \Sigma_{t}^{\prime}\left(\mathbf{R}^{d}\right) \\
& \hookrightarrow \mathcal{S}_{s}^{\prime}\left(\mathbf{R}^{d}\right) \hookrightarrow \Sigma_{s}^{\prime}\left(\mathbf{R}^{d}\right) \hookrightarrow \mathcal{S}_{1 / 2}^{\prime}\left(\mathbf{R}^{d}\right), \quad \frac{1}{2}<s<t .
\end{aligned}
$$

Here and in what follows we use the notation $A \hookrightarrow B$ when the topological spaces $A$ and $B$ satisfy $A \subseteq B$ with continuous embeddings.

A convenient family of functions concerns the Hermite functions

$$
h_{\alpha}(x)=\pi^{-\frac{d}{4}}(-1)^{|\alpha|}\left(2^{|\alpha|} \alpha !\right)^{-\frac{1}{2}} e^{\frac{|x|^{2}}{2}}\left(\partial^{\alpha} e^{-|x|^{2}}\right), \quad \alpha \in \mathbf{N}^{d} .
$$

The set of Hermite functions on $\mathbf{R}^{d}$ is an orthonormal basis for $L^{2}\left(\mathbf{R}^{d}\right)$. It is also a basis for the Schwartz space and its distribution space, and for any $\Sigma_{s}$ when $s>\frac{1}{2}$, $\mathcal{S}_{s}$ when $s \geqslant \frac{1}{2}$ and their distribution spaces. They are also eigenfunctions to the Harmonic oscillator $H=H_{d} \equiv|x|^{2}-\Delta$ and to the Fourier transform $\mathscr{F}$, given by

$$
(\mathscr{F} f)(\xi)=\widehat{f}(\xi) \equiv(2 \pi)^{-\frac{d}{2}} \int_{\mathbf{R}^{d}} f(x) e^{-i\langle x, \xi\rangle} d x, \quad \xi \in \mathbf{R}^{d}
$$

when $f \in L^{1}\left(\mathbf{R}^{d}\right)$. Here $\langle\cdot, \cdot\rangle$ denotes the usual scalar product on $\mathbf{R}^{d}$. In fact, we have

$$
H_{d} h_{\alpha}=(2|\alpha|+d) h_{\alpha}
$$

The Fourier transform $\mathscr{F}$ extends uniquely to homeomorphisms on $\mathscr{S}^{\prime}\left(\mathbf{R}^{d}\right)$, $\mathcal{S}_{s}^{\prime}\left(\mathbf{R}^{d}\right)$ and on $\Sigma_{s}^{\prime}\left(\mathbf{R}^{d}\right)$. Furthermore, $\mathscr{F}$ restricts to homeomorphisms on $\mathscr{S}\left(\mathbf{R}^{d}\right)$, $\mathcal{S}_{s}\left(\mathbf{R}^{d}\right)$ and on $\Sigma_{s}\left(\mathbf{R}^{d}\right)$, and to a unitary operator on $L^{2}\left(\mathbf{R}^{d}\right)$. Similar facts hold true when the Fourier transform is replaced by a partial Fourier transform.

Gelfand-Shilov spaces and their distribution spaces can also be characterized by estimates of short-time Fourier transform, (see e.g. [14, 21, 26]). More precisely, let $\phi \in \mathscr{S}\left(\mathbf{R}^{d}\right)$ be fixed. Then the short-time Fourier transform $V_{\phi} f$ of $f \in \mathscr{S}^{\prime}\left(\mathbf{R}^{d}\right)$ with respect to the window function $\phi$ is the Schwartz distribution on $\mathbf{R}^{2 d}$, defined by

$$
V_{\phi} f(x, \xi)=\mathscr{F}(f \overline{\phi(\cdot-x)})(\xi), \quad x, \xi \in \mathbf{R}^{d} .
$$

If $f, \phi \in \mathscr{S}\left(\mathbf{R}^{d}\right)$, then it follows that

$$
V_{\phi} f(x, \xi)=(2 \pi)^{-\frac{d}{2}} \int_{\mathbf{R}^{d}} f(y) \overline{\phi(y-x)} e^{-i\langle y, \xi\rangle} d y, \quad x, \xi \in \mathbf{R}^{d} .
$$

By [25, Theorem 2.3] it follows that the definition of the map $(f, \phi) \mapsto V_{\phi} f$ from $\mathscr{S}\left(\mathbf{R}^{d}\right) \times \mathscr{S}\left(\mathbf{R}^{d}\right)$ to $\mathscr{S}\left(\mathbf{R}^{2 d}\right)$ is uniquely extendable to a continuous map from 
$\mathcal{S}_{s}^{\prime}\left(\mathbf{R}^{d}\right) \times \mathcal{S}_{s}^{\prime}\left(\mathbf{R}^{d}\right)$ to $\mathcal{S}_{s}^{\prime}\left(\mathbf{R}^{2 d}\right)$, and restricts to a continuous map from $\mathcal{S}_{s}\left(\mathbf{R}^{d}\right) \times \mathcal{S}_{s}\left(\mathbf{R}^{d}\right)$ to $\mathcal{S}_{s}\left(\mathbf{R}^{2 d}\right)$. The same conclusion holds with $\Sigma_{s}$ in place of $\mathcal{S}_{s}$, at each place.

In the following propositions we give characterizations of Gelfand-Shilov spaces and their distribution spaces in terms of estimates of the short-time Fourier transform. We omit the proof since the first part follows from [14, Theorem 2.7]) and the second part from [26, Proposition 2.2]. See also [8] for related results.

Proposition 1.1. Let $s \geqslant \frac{1}{2}\left(s>\frac{1}{2}\right), \phi \in \mathcal{S}_{s}\left(\mathbf{R}^{d}\right) \backslash 0\left(\phi \in \Sigma_{s}\left(\mathbf{R}^{d}\right) \backslash 0\right)$ and let $f$ be a Gelfand-Shilov distribution on $\mathbf{R}^{d}$. Then the following is true:

(1) $f \in \mathcal{S}_{s}\left(\mathbf{R}^{d}\right)\left(f \in \Sigma_{s}\left(\mathbf{R}^{d}\right)\right)$, if and only if

$$
\left|V_{\phi} f(x, \xi)\right| \lesssim e^{-r\left(|x|^{\frac{1}{s}}+|\xi|^{\frac{1}{s}}\right)}, \quad x, \xi \in \mathbf{R}^{d},
$$

for some $r>0$ (for every $r>0$ ).

(2) $f \in \mathcal{S}_{s}^{\prime}\left(\mathbf{R}^{d}\right)\left(f \in \Sigma_{s}^{\prime}\left(\mathbf{R}^{d}\right)\right)$, if and only if

$$
\left|V_{\phi} f(x, \xi)\right| \lesssim e^{r\left(|x|^{\frac{1}{s}}+|\xi|^{\frac{1}{s}}\right)}, \quad x, \xi \in \mathbf{R}^{d}
$$

for every $r>0$ (for some $r>0$ ).

1.3. Spaces of Hermite series and power series expansions. Next we recall the definitions of topological vector spaces of Hermite series expansions, given in [26]. As in [26], it is convenient to use suitable extensions of $\mathbf{R}_{+}$when indexing our spaces.

Definition 1.2. The sets $\mathbf{R}_{b}$ and $\overline{\mathbf{R}_{b}}$ are given by

$$
\mathbf{R}_{b}=\mathbf{R}_{+} \bigcup_{\sigma>0}\left\{b_{\sigma}\right\} \quad \text { and } \quad \overline{\mathbf{R}_{b}}=\mathbf{R}_{b} \bigcup\{0\} .
$$

Moreover, beside the usual ordering in $\mathbf{R}$, the elements $b_{\sigma}$ in $\mathbf{R}_{b}$ and $\overline{\mathbf{R}_{b}}$ are ordered by the relations $x_{1}<b_{\sigma_{1}}<b_{\sigma_{2}}<x_{2}$, when $\sigma_{1}, \sigma_{2}, x_{1}$ and $x_{2}$ are positive real numbers such that $x_{1}<\frac{1}{2}, x_{2} \geqslant \frac{1}{2}$ and $\sigma_{1}<\sigma_{2}$.

Definition 1.3. Let $p \in[1, \infty], s \in \mathbf{R}_{b}, r \in \mathbf{R}, \vartheta$ be a weight on $\mathbf{N}^{d}$, and let

$$
\vartheta_{r, s}(\alpha) \equiv\left\{\begin{array}{lll}
e^{r|\alpha|^{\frac{1}{2 s}}}, & \text { when } & s \in \mathbf{R}_{+}, \\
r^{|\alpha|}(\alpha !)^{\frac{1}{2 \sigma}}, & \text { when } & s=b_{\sigma},
\end{array} \quad \alpha \in \mathbf{N}^{d} .\right.
$$

Then,

(1) $\ell_{0}^{\prime}\left(\mathbf{N}^{d}\right)$ is the set of all sequences $\left\{c_{\alpha}\right\}_{\alpha \in \mathbf{N}^{d}} \subseteq \mathbf{C}$ on $\mathbf{N}^{d}$;

(2) $\ell_{0,0}\left(\mathbf{N}^{d}\right) \equiv\{0\}$, and $\ell_{0}\left(\mathbf{N}^{d}\right)$ is the set of all sequences $\left\{c_{\alpha}\right\}_{\alpha \in \mathbf{N}^{d}} \subseteq \mathbf{C}$ such that $c_{\alpha} \neq 0$ for at most finite numbers of $\alpha$;

(3) $\ell_{[\vartheta]}^{p}\left(\mathbf{N}^{d}\right)$ is the Banach space which consists of all sequences $\left\{c_{\alpha}\right\}_{\alpha \in \mathbf{N}^{d}} \subseteq \mathbf{C}$ such that

$$
\left\|\left\{c_{\alpha}\right\}_{\alpha \in \mathbf{N}^{d}}\right\|_{\ell_{[\vartheta]}^{p}} \equiv\left\|\left\{c_{\alpha} \vartheta(\alpha)\right\}_{\alpha \in \mathbf{N}^{d}}\right\|_{\ell^{p}}<\infty ;
$$

(4) $\ell_{0, s}\left(\mathbf{N}^{d}\right) \equiv \bigcap_{r>0} \ell_{\left[\vartheta_{r, s}\right]}^{p}\left(\mathbf{N}^{d}\right)$ and $\ell_{s}\left(\mathbf{N}^{d}\right) \equiv \bigcup_{r>0} \ell_{\left[\vartheta_{r, s}\right]}^{p}\left(\mathbf{N}^{d}\right)$, with projective respective inductive limit topologies of $\ell_{\left[\vartheta_{r, s}\right]}^{p}\left(\mathbf{N}^{d}\right)$ with respect to $r>0$;

(5) $\ell_{0, s}^{\prime}\left(\mathbf{N}^{d}\right) \equiv \bigcup_{r>0} \ell_{\left[1 / \vartheta_{r, s}\right]}^{p}\left(\mathbf{N}^{d}\right)$ and $\ell_{s}^{\prime}\left(\mathbf{N}^{d}\right) \equiv \bigcap_{r>0} \ell_{\left[1 / \vartheta_{r, s}\right]}^{p}\left(\mathbf{N}^{d}\right)$, with inductive respective projective limit topologies of $\ell_{\left[1 / \vartheta_{r, s}\right]}^{p}\left(\mathbf{N}^{d}\right)$ with respect to $r>0$.

Let $p \in[1, \infty]$, and let $\Omega_{N}$ be the set of all $\alpha \in \mathbf{N}^{d}$ such that $|\alpha| \leqslant N$. Then the topology of $\ell_{0}\left(\mathbf{N}^{d}\right)$ is defined by the inductive limit topology of the sets

$$
\left\{\left\{c_{\alpha}\right\}_{\alpha \in \mathbf{N}^{d}} \in \ell_{0}^{\prime}\left(\mathbf{N}^{d}\right) ; c_{\alpha}=0 \text { when } \alpha \notin \Omega_{N}\right\}
$$


with respect to $N \geqslant 0$, and whose topology is given through the semi-norms

$$
\left\{c_{\alpha}\right\}_{\alpha \in \mathbf{N}^{d}} \mapsto\left\|\left\{c_{\alpha}\right\}_{|\alpha| \leqslant N}\right\|_{\ell^{p}\left(\Omega_{N}\right)},
$$

It is clear that these topologies are independent of $p$. Furthermore, the topology of $\ell_{0}^{\prime}\left(\mathbf{N}^{d}\right)$ is defined by the semi-norms (1.9). It follows that $\ell_{0}^{\prime}\left(\mathbf{N}^{d}\right)$ is a Fréchet space, and that its topology as well as the topologies of the spaces in Definition 1.3. (4) and (5) are independent of $p$.

Next we introduce spaces of formal Hermite series expansions

$$
f=\sum_{\alpha \in \mathbf{N}^{d}} c_{\alpha} h_{\alpha}, \quad\left\{c_{\alpha}\right\}_{\alpha \in \mathbf{N}^{d}} \in \ell_{0}^{\prime}\left(\mathbf{N}^{d}\right)
$$

and power series expansions

$$
F=\sum_{\alpha \in \mathbf{N}^{d}} c_{\alpha} e_{\alpha}, \quad\left\{c_{\alpha}\right\}_{\alpha \in \mathbf{N}^{d}} \in \ell_{0}^{\prime}\left(\mathbf{N}^{d}\right)
$$

which correspond to

$$
\ell_{0, s}\left(\mathbf{N}^{d}\right), \quad \ell_{s}\left(\mathbf{N}^{d}\right), \quad \ell_{s}^{\prime}\left(\mathbf{N}^{d}\right) \quad \text { and } \quad \ell_{0, s}^{\prime}\left(\mathbf{N}^{d}\right)
$$

Here

$$
e_{\alpha}(z) \equiv \frac{z^{\alpha}}{\sqrt{\alpha !}}, \quad z \in \mathbf{C}^{d}, \alpha \in \mathbf{N}^{d}
$$

We consider the mappings

$$
T_{\mathcal{H}}:\left\{c_{\alpha}\right\}_{\alpha \in \mathbf{N}^{d}} \mapsto \sum_{\alpha \in \mathbf{N}^{d}} c_{\alpha} h_{\alpha} \quad \text { and } \quad T_{\mathcal{A}}:\left\{c_{\alpha}\right\}_{\alpha \in \mathbf{N}^{d}} \mapsto \sum_{\alpha \in \mathbf{N}^{d}} c_{\alpha} e_{\alpha}
$$

between sequences, and formal Hermite series and power series expansions.

Definition 1.4. If $s \in \overline{\mathbf{R}_{b}}$, then

$$
\mathcal{H}_{0, s}\left(\mathbf{R}^{d}\right), \quad \mathcal{H}_{s}\left(\mathbf{R}^{d}\right), \quad \mathcal{H}_{s}^{\prime}\left(\mathbf{R}^{d}\right) \quad \text { and } \quad \mathcal{H}_{0, s}^{\prime}\left(\mathbf{R}^{d}\right),
$$

and

$$
\mathcal{A}_{0, s}\left(\mathbf{C}^{d}\right), \quad \mathcal{A}_{s}\left(\mathbf{C}^{d}\right), \quad \mathcal{A}_{s}^{\prime}\left(\mathbf{C}^{d}\right) \quad \text { and } \quad \mathcal{A}_{0, s}^{\prime}\left(\mathbf{C}^{d}\right),
$$

are the images of $T_{\mathcal{H}}$ and $T_{\mathcal{A}}$ respectively in (1.14) of corresponding spaces in (1.12). The topologies of the spaces in (1.15) and (1.16) are inherited from the corresponding spaces in (1.12).

Since locally absolutely convergent power series expansions can be identified with entire functions, several of the spaces in (1.16) are identified with topological vector spaces contained in $A\left(\mathbf{C}^{d}\right)$ (see Theorem 1.9 below and the introduction). Here $A\left(\Omega_{0}\right)$ is the set of all (complex valued) functions which are analytic in $\Omega_{0}$. (For $\Omega_{0} \subseteq \mathbf{C}^{d}$, $A\left(\Omega_{0}\right)=\bigcup A(\Omega)$, where the union is taken over all open $\Omega \subseteq \mathbf{C}^{d}$ which contain $\Omega_{0}$. We also set $A_{d}(\{0\})=A(\{0\})$ when $0 \in \mathbf{C}^{d}$.)

We recall that $f \in \mathscr{S}\left(\mathbf{R}^{d}\right)$ if and only if it can be written as (1.10) such that

$$
\left|c_{\alpha}\right| \lesssim\langle\alpha\rangle^{-N}
$$


for every $N \geqslant 0$ (cf. e.g. [19]). In particular it follows from the definitions that the inclusions

$$
\begin{aligned}
\mathcal{H}_{0}\left(\mathbf{R}^{d}\right) \hookrightarrow & \mathcal{H}_{0, s}\left(\mathbf{R}^{d}\right) \hookrightarrow \\
\hookrightarrow \mathscr{H}\left(\mathbf{R}^{d}\right) \hookrightarrow & \mathscr{S}^{\prime}\left(\mathbf{R}^{d}\right) \hookrightarrow \mathcal{H}_{0, t}\left(\mathbf{R}^{d}\right) \\
& \hookrightarrow \mathcal{H}_{0, t}^{\prime}\left(\mathbf{R}^{d}\right) \hookrightarrow \mathcal{H}_{s}^{\prime}\left(\mathbf{R}^{d}\right) \\
& \hookrightarrow \mathcal{H}_{0, s}^{\prime}\left(\mathbf{R}^{d}\right) \hookrightarrow \mathcal{H}_{0}^{\prime}\left(\mathbf{R}^{d}\right), \quad \text { when } s, t \in \mathbf{R}_{b}, s<t
\end{aligned}
$$

are dense.

Remark 1.5. By the definition it follows that $T_{\mathcal{H}}$ in (1.14) is a homeomorphism between any of the spaces in (1.12) and corresponding space in (1.15), and that $T_{\mathcal{A}}$ in (1.14) is a homeomorphism between any of the spaces in (1.12) and corresponding space in (1.16).

The next results give some characterizations of $\mathcal{H}_{s}\left(\mathbf{R}^{d}\right)$ and $\mathcal{H}_{0, s}\left(\mathbf{R}^{d}\right)$ when $s$ is a non-negative real number.

Proposition 1.6. Let $0 \leqslant s \in \mathbf{R}$ and let $f \in \mathcal{H}_{0}^{\prime}\left(\mathbf{R}^{d}\right)$. Then $f \in \mathcal{H}_{s}\left(\mathbf{R}^{d}\right)$ $\left(f \in \mathcal{H}_{0, s}\left(\mathbf{R}^{d}\right)\right)$, if and only if $f \in C^{\infty}\left(\mathbf{R}^{d}\right)$ and satisfies

$$
\left\|H_{d}^{N} f\right\|_{L^{\infty}} \lesssim h^{N} N !^{2 s},
$$

for some $h>0$ (every $h>0$ ). Moreover, it holds

$$
\begin{array}{llll}
\mathcal{H}_{s}\left(\mathbf{R}^{d}\right)=\mathcal{S}_{s}\left(\mathbf{R}^{d}\right) \neq\{0\}, & \mathcal{H}_{0, s}\left(\mathbf{R}^{d}\right)=\Sigma_{s}\left(\mathbf{R}^{d}\right) \neq\{0\} & \text { when } & s \in\left(\frac{1}{2}, \infty\right), \\
\mathcal{H}_{s}\left(\mathbf{R}^{d}\right)=\mathcal{S}_{s}\left(\mathbf{R}^{d}\right) \neq\{0\}, & \mathcal{H}_{0, s}\left(\mathbf{R}^{d}\right) \neq \Sigma_{s}\left(\mathbf{R}^{d}\right)=\{0\} & \text { when } & s=\frac{1}{2}, \\
\mathcal{H}_{s}\left(\mathbf{R}^{d}\right) \neq \mathcal{S}_{s}\left(\mathbf{R}^{d}\right)=\{0\}, & \mathcal{H}_{0, s}\left(\mathbf{R}^{d}\right) \neq \Sigma_{s}\left(\mathbf{R}^{d}\right)=\{0\} & \text { when } & s \in\left(0, \frac{1}{2}\right), \\
\mathcal{H}_{s}\left(\mathbf{R}^{d}\right) \neq \mathcal{S}_{s}\left(\mathbf{R}^{d}\right)=\{0\}, & \mathcal{H}_{0, s}\left(\mathbf{R}^{d}\right)=\Sigma_{s}\left(\mathbf{R}^{d}\right)=\{0\} & \text { when } & s=0 .
\end{array}
$$

We refer to [26] for the proof of Proposition 1.6.

Due to the pioneering investigations related to Proposition 1.6 by Pilipović in $[17,18]$, we call the spaces $\mathcal{H}_{s}\left(\mathbf{R}^{d}\right)$ and $\mathcal{H}_{0, s}\left(\mathbf{R}^{d}\right)$ Pilipović spaces of Roumieu and Beurling types, respectively. In fact, in the restricted case $s \geqslant \frac{1}{2}$, Proposition 1.6 was proved already in $[17,18]$.

Later on it will also be convenient for us to have the following definition. Here we let $F\left(z_{2}, \bar{z}_{1}\right)$ and $F\left(\bar{z}_{2}, z_{1}\right)$ be the formal power series

$$
\sum c\left(\alpha_{2}, \alpha_{1}\right) e_{\alpha_{2}}\left(z_{2}\right) e_{\alpha_{1}}\left(\bar{z}_{1}\right) \text { and } \sum c\left(\alpha_{2}, \alpha_{1}\right) e_{\alpha_{2}}\left(\bar{z}_{2}\right) e_{\alpha_{1}}\left(z_{1}\right)
$$

respectively, when $F\left(z_{2}, z_{1}\right)$ is the formal power series

$$
\sum c\left(\alpha_{2}, \alpha_{1}\right) e_{\alpha_{2}}\left(z_{2}\right) e_{\alpha_{1}}\left(z_{1}\right) \text {. }
$$

Here $z_{j} \in \mathbf{C}^{d_{j}}, j=1,2$, and the sums should be taken over all $\left(\alpha_{2}, \alpha_{1}\right) \in \mathbf{N}^{d_{2}} \times \mathbf{N}^{d_{1}}$.

Definition 1.7. Let $d=d_{2}+d_{1}, s \in \overline{\mathbf{R}_{b}}, \Theta_{C, 1}$ and $\Theta_{C, 2}$ be the operators

$$
\left(\Theta_{C, 1} F\right)\left(z_{2}, z_{1}\right)=F\left(z_{2}, \bar{z}_{1}\right) \text { and }\left(\Theta_{C, 2} F\right)\left(z_{2}, z_{1}\right)=F\left(\bar{z}_{2}, z_{1}\right)
$$

between formal power series in (1.19) and (1.20), $z_{j} \in \mathbf{C}^{d_{j}}, j=1,2$. Then

$$
\hat{\mathcal{A}}_{0, s}\left(\mathbf{C}^{d_{2}} \times \mathbf{C}^{d_{1}}\right), \quad \hat{\mathcal{A}}_{s}\left(\mathbf{C}^{d_{2}} \times \mathbf{C}^{d_{1}}\right), \quad \hat{\mathcal{A}}_{s}^{\prime}\left(\mathbf{C}^{d_{2}} \times \mathbf{C}^{d_{1}}\right), \quad \hat{\mathcal{A}}_{0, s}^{\prime}\left(\mathbf{C}^{d_{2}} \times \mathbf{C}^{d_{1}}\right)
$$

are the images of (1.16) under $\Theta_{C, 1}$, and $\widehat{A}\left(\mathbf{C}^{d_{2}} \times \mathbf{C}^{d_{1}}\right)$ and $\widehat{A}_{d_{2}, d_{1}}(\{0\})=\widehat{A}_{d_{2}+d_{1}}(\{0\})$ are the images of $A\left(\mathbf{C}^{d}\right)$ and $A_{d_{2}+d_{1}}(\{0\})$ respectively under $\Theta_{C, 1}$. The topologies of the spaces in (1.21), $\widehat{A}\left(\mathbf{C}^{d_{2}} \times \mathbf{C}^{d_{1}}\right)$ and $\widehat{A}_{d_{2}, d_{1}}(\{0\})$ are inherited from the topologies in the spaces (1.16), $A\left(\mathbf{C}^{d}\right)$ and $A_{d}(\{0\})$, respectively. 
Remark 1.8. By letting $d_{2}=d$ and $d_{1}=0$, it follows that $A\left(\mathbf{C}^{d}\right)$ and the spaces in (1.16) can be considered as special cases of $\widehat{A}\left(\mathbf{C}^{d_{2}} \times \mathbf{C}^{d_{1}}\right)$ and the spaces in (1.21). Since $\mathcal{A}_{b_{1}}^{\prime}\left(\mathbf{C}^{d}\right)=A\left(\mathbf{C}^{d}\right)$ and $\mathcal{A}_{0, b_{1}}^{\prime}\left(\mathbf{C}^{d}\right)=A_{d}(\{0\})$, it follows that

$$
\begin{aligned}
\hat{\mathcal{A}}_{b_{1}}^{\prime}\left(\mathbf{C}^{d_{2}} \times \mathbf{C}^{d_{1}}\right) & =\hat{A}\left(\mathbf{C}^{d_{2}} \times \mathbf{C}^{d_{1}}\right), \\
\widehat{\mathcal{A}}_{0, b_{1}}^{\prime}\left(\mathbf{C}^{d_{2}} \times \mathbf{C}^{d_{1}}\right) & =\widehat{A}_{d_{2}+d_{1}}(\{0\}) .
\end{aligned}
$$

The following results are now immediate consequences of Theorems 4.1, 4.2, 5.2 and 5.3 in [26] and Definition 1.7. Here let

$$
\kappa_{1, r, s}(z)= \begin{cases}e^{r(\log \langle z\rangle) \frac{1}{1-2 s}}, & s<\frac{1}{2} \\ e^{r|z|^{\frac{2 \sigma}{\sigma+1}}}, & s=b_{\sigma}, \sigma>0 \\ e^{\frac{|z|^{2}}{2}-r|z|^{\frac{1}{s}}}, & s \geqslant \frac{1}{2}\end{cases}
$$

and

$$
\kappa_{2, r, s}(z)= \begin{cases}e^{r|z|^{\frac{2 \sigma}{\sigma-1}}}, & s=b_{\sigma}, \sigma>1, \\ e^{\frac{|z|^{2}}{2}+r|z|^{\frac{1}{s}}}, & s \geqslant \frac{1}{2},\end{cases}
$$

Theorem 1.9. Let $s_{1}, s_{2} \in \mathbf{R}_{b}$ be such that $s_{2}>b_{1}$, and let $\kappa_{1, r, s}$ and $\kappa_{2, r, s}$ be given by (1.23) and (1.24) respectively, when $r>0$. Then the following is true:

(1) $\hat{\mathcal{A}}_{s_{1}}\left(\mathbf{C}^{d_{2}} \times \mathbf{C}^{d_{1}}\right)\left(\hat{\mathcal{A}}_{0, s_{1}}\left(\mathbf{C}^{d_{2}} \times \mathbf{C}^{d_{1}}\right)\right)$ consists of all $K \in \widehat{A}\left(\mathbf{C}^{d_{2}} \times \mathbf{C}^{d_{1}}\right)$ such that $|K| \lesssim \kappa_{1, r, s_{1}}$ for some $r>0$ (for every $0<r<\frac{1}{2}$ ).

(2) $\hat{\mathcal{A}}_{s_{2}}^{\prime}\left(\mathbf{C}^{d_{2}} \times \mathbf{C}^{d_{1}}\right)\left(\widehat{\mathcal{A}}_{0, s_{2}}^{\prime}\left(\mathbf{C}^{d_{2}} \times \mathbf{C}^{d_{1}}\right)\right)$ consists of all $K \in \widehat{A}\left(\mathbf{C}^{d_{2}} \times \mathbf{C}^{d_{1}}\right)$ such that $|K| \lesssim \kappa_{2, r, s_{2}}$ for every $r>0$ (for some $r>0$ ).

By Remark 1.8 it follows that Theorem 1.9 remains true after the spaces in (1.21) are replaced by corresponding spaces in (1.16).

1.4. Modulation spaces. Before giving the definition of a broad family of modulation spaces, we make a review of mixed normed spaces of Lebesgue types, adapted to suitable bases of the Euclidean space $\mathbf{R}^{d}$. Let $E$ be the ordered basis $\left\{e_{1}, \ldots, e_{d}\right\}$ of $\mathbf{R}^{d}$. Then the ordered basis $E^{\prime}=\left\{e_{1}^{\prime}, \ldots, e_{d}^{\prime}\right\}$ (the dual basis of $E$ ) satisfies

$$
\left\langle e_{j}, e_{k}^{\prime}\right\rangle=2 \pi \delta_{j k} \quad \text { for every } \quad j, k=1, \ldots, d .
$$

The corresponding parallelepiped, lattice, dual parallelepiped and dual lattice are given by

$$
\begin{aligned}
\kappa(E) & =\left\{x_{1} e_{1}+\cdots+x_{d} e_{d} ;\left(x_{1}, \ldots, x_{d}\right) \in \mathbf{R}^{d}, 0 \leqslant x_{k} \leqslant 1, k=1, \ldots, d\right\}, \\
\Lambda_{E} & =\left\{j_{1} e_{1}+\cdots+j_{d} e_{d} ;\left(j_{1}, \ldots, j_{d}\right) \in \mathbf{Z}^{d}\right\}, \\
\kappa\left(E^{\prime}\right) & =\left\{\xi_{1} e_{1}^{\prime}+\cdots+\xi_{d} e_{d}^{\prime} ;\left(\xi_{1}, \ldots, \xi_{d}\right) \in \mathbf{R}^{d}, 0 \leqslant \xi_{k} \leqslant 1, k=1, \ldots, d\right\},
\end{aligned}
$$

and

$$
\Lambda_{E}^{\prime}=\Lambda_{E^{\prime}}=\left\{\iota_{1} e_{1}^{\prime}+\cdots+\iota_{d} e_{d}^{\prime} ;\left(\iota_{1}, \ldots, \iota_{d}\right) \in \mathbf{Z}^{d}\right\},
$$

respectively. Note here that the Fourier analysis with respect to general biorthogonal bases has recently been developed in [20].

We observe that there is a matrix $T_{E}$ such that $e_{1}, \ldots, e_{d}$ and $e_{1}^{\prime}, \ldots, e_{d}^{\prime}$ are the images of the standard basis under $T_{E}$ and $T_{E^{\prime}}=2 \pi\left(T_{E}^{-1}\right)^{t}$, respectively.

In the following we let

$$
\max (\boldsymbol{q})=\max \left(q_{1}, \ldots, q_{d}\right) \quad \text { and } \quad \min (\boldsymbol{q})=\min \left(q_{1}, \ldots, q_{d}\right)
$$


when $\boldsymbol{q}=\left(q_{1}, \ldots, q_{d}\right) \in[1, \infty]^{d}$.

Definition 1.10. Let $E$ be an ordered basis of $\mathbf{R}^{d}$ and $\boldsymbol{p}=\left(p_{1}, \ldots, p_{d}\right) \in[1, \infty]^{d}$. If $f \in L_{\mathrm{loc}}^{1}\left(\mathbf{R}^{d}\right)$, then $\|f\|_{L_{E}^{p}}$ is defined by

$$
\|f\|_{L_{E}^{p}} \equiv\left\|g_{d-1}\right\|_{L^{p}(\mathbf{R})}
$$

where $g_{k}\left(z_{k}\right), z_{k} \in \mathbf{R}^{d-k}, k=0, \ldots, d-1$, are inductively defined as

$$
g_{0}\left(x_{1}, \ldots, x_{d}\right) \equiv\left|f\left(x_{1} e_{1}+\cdots+x_{d} e_{d}\right)\right|, \quad\left(x_{1}, \ldots, x_{d}\right) \in \mathbf{R}^{d},
$$

and

$$
g_{k}\left(z_{k}\right) \equiv\left\|g_{k-1}\left(\cdot, z_{k}\right)\right\|_{L^{p_{k}(\mathbf{R})}}, \quad z_{k} \in \mathbf{R}^{d-k}, \quad k=1, \ldots, d-1 .
$$

The space $L_{E}^{p}\left(\mathbf{R}^{d}\right)$ consists of all $f \in L_{\text {loc }}^{1}\left(\mathbf{R}^{d}\right)$ such that $\|f\|_{L_{E}^{p}}$ is finite, and is called E-split Lebesgue space (with respect to $\boldsymbol{p}$ ).

Next we discuss suitable conditions for bases in the phase space $\mathbf{R}^{2 d}$. We let $\sigma(X, Y)$ be the standard symplectic form on the phase space, given by

$$
\sigma(X, Y)=\langle y, \xi\rangle-\langle x, \eta\rangle, \quad X=(x, \xi) \in \mathbf{R}^{2 d}, Y=(y, \eta) \in \mathbf{R}^{2 d} .
$$

We notice that if

$$
\left\{e_{1}, \ldots, e_{d}, \varepsilon_{1}, \ldots, \varepsilon_{d}\right\}
$$

is the standard basis of $\mathbf{R}^{2 d}$, then

$$
\sigma\left(e_{j}, e_{k}\right)=0, \quad \sigma\left(e_{j}, \varepsilon_{k}\right)=-\delta_{j, k}, \quad \text { and } \quad \sigma\left(\varepsilon_{j}, \varepsilon_{k}\right)=0,
$$

when $j, k \in\{1, \ldots, d\}$. More generally, a basis in (1.25) for the phase space $\mathbf{R}^{2 d}$ is called symplectic if (1.26) holds. A symplectic basis (1.25) for $\mathbf{R}^{2 d}$ is called phase split if $e_{1}, \ldots, e_{d}$ and $\varepsilon_{1}, \ldots, \varepsilon_{d}$ span

$$
\left\{(x, 0) \in \mathbf{R}^{2 d} ; x \in \mathbf{R}^{d}\right\} \quad \text { and } \quad\left\{(0, \xi) \in \mathbf{R}^{2 d} ; \xi \in \mathbf{R}^{d}\right\},
$$

respectively.

Next we give the definition of our class of modulation spaces.

Definition 1.11. Let $E$ be an ordered basis for $\mathbf{R}^{2 d}, \boldsymbol{p} \in[1, \infty]^{2 d}, \phi(x)=$ $\pi^{-\frac{d}{4}} e^{-\frac{1}{2} \cdot|x|^{2}}$ and let $\omega$ be a weight on $\mathbf{R}^{2 d}$. Then the modulation space $M_{E,(\omega)}^{p}\left(\mathbf{R}^{d}\right)$ consists of all $f \in \mathcal{H}_{b_{1}}^{\prime}\left(\mathbf{R}^{d}\right)$ such that

$$
\|f\|_{M_{E,(\omega)}^{p}} \equiv\left\|V_{\phi} f \cdot \omega\right\|_{L_{E}^{p}}
$$

is finite.

We remark that if $\phi(x)=\pi^{-\frac{d}{4}} e^{-\frac{1}{2} \cdot|x|^{2}}$ and $f \in \mathcal{H}_{b_{1}}^{\prime}\left(\mathbf{R}^{d}\right)$, then $(x, \xi) \mapsto V_{\phi} f(x, \xi)$ is a smooth function (cf. [26]). Furthermore, by [26, Theorem 4.8] we get the following. The proof is omitted.

Proposition 1.12. Let $E$ be an ordered basis for $\mathbf{R}^{2 d}, \boldsymbol{p} \in[1, \infty]^{2 d}$ and let $\omega$ be a weight on $\mathbf{R}^{2 d}$. Then $M_{E,(\omega)}^{p}\left(\mathbf{R}^{d}\right)$ is a Banach space with norm given by (1.27).

If the weight $\omega$ in Definition 1.11 is a moderate weight, then we can say more concerning $M_{E,(\omega)}^{p}\left(\mathbf{R}^{d}\right)$. In what follows we let $p^{\prime} \in[1, \infty]$ be the conjugate exponent of $p \in[1, \infty]$, i.e. $\frac{1}{p}+\frac{1}{p^{\prime}}=1$.

Proposition 1.13. Let $E$ be an ordered basis for $\mathbf{R}^{2 d}, \boldsymbol{p} \in[1, \infty]^{2 d}$ and let $\omega, v \in \mathscr{P}_{E}\left(\mathbf{R}^{2 d}\right)$ be such that $\omega$ is $v$-moderate. Then the following is true: 
(1) $\Sigma_{1}\left(\mathbf{R}^{d}\right) \hookrightarrow M_{E,(\omega)}^{p}\left(\mathbf{R}^{d}\right) \hookrightarrow \Sigma_{1}^{\prime}\left(\mathbf{R}^{d}\right)$. If in addition $\max (\boldsymbol{p})<\infty$, then $\Sigma_{1}\left(\mathbf{R}^{d}\right)$ is dense in $M_{E,(\omega)}^{p}\left(\mathbf{R}^{d}\right)$;

(2) if $\phi \in M_{(v)}^{1}\left(\mathbf{R}^{d}\right) \backslash\{0\}$ and $f \in \Sigma_{1}^{\prime}\left(\mathbf{R}^{d}\right)$, then $f \in M_{E,(\omega)}^{p}\left(\mathbf{R}^{d}\right)$, if and only if the right-hand side of (1.27) is finite. Furthermore, different choices of $\phi \in M_{(v)}^{1}\left(\mathbf{R}^{d}\right) \backslash\{0\}$ in (1.27) give rise to equivalent norms;

(3) $M_{E,(\omega)}^{p}\left(\mathbf{R}^{d}\right)$ increases with $p_{1}, \ldots, p_{2 d}$ and decreases with $\omega$;

(4) if $\boldsymbol{p}^{\prime}=\left(p_{1}^{\prime}, \ldots, p_{2 d}^{\prime}\right)$, then the restriction of the $L^{2}\left(\mathbf{R}^{d}\right)$ scalar product $(\cdot, \cdot)$ to $\Sigma_{1}\left(\mathbf{R}^{d}\right)$ is uniquely extendable to a (semi-conjugate) duality between $M_{E,(\omega)}^{p}\left(\mathbf{R}^{d}\right)$ and $M_{E,(1 / \omega)}^{p^{\prime}}\left(\mathbf{R}^{d}\right)$. If in addition $\max (\boldsymbol{p})<\infty$, then the dual of $M_{E,(\omega)}^{p}\left(\mathbf{R}^{d}\right)$ can be identified by $M_{E,(1 / \omega)}^{p^{\prime}}\left(\mathbf{R}^{d}\right)$ through the form $(\cdot, \cdot)$.

Proposition 1.13 follows by similar arguments as in Chapters 11 and 12 in [12] (see also $[25,26]$ ).

Remark 1.14. In some sense, the variable $x$ at the weight $\omega(x, \xi)$ in the definition of modulation spaces quantify decay and possible growth properties for the involved functions or distributions. In the same way the variable $\xi$ quantify regularity or possible lack of regularity for the involved functions or distributions.

By the analysis in [26] it follows that there are no bounds on how fast $V_{\phi} f$ may grow or decay at infinity when $\phi(x)=\pi^{-\frac{d}{4}} e^{-\frac{1}{2} \cdot|x|^{2}}$ is fixed, $x \in \mathbf{R}^{d}$, and $f$ is taken in the class $\mathcal{H}_{b_{1}}^{\prime}\left(\mathbf{R}^{d}\right)$. Since weights in $\mathscr{P}_{E}\left(\mathbf{R}^{2 d}\right)$ are bounded by exponential functions, the restrictions of the weights in Proposition 1.13 are significantly stronger compared to what is the case in Proposition 1.12. A question here concerns wether it is possible to extend parts of Proposition 1.13 to larger weight classes than $\mathscr{P}_{E}\left(\mathbf{R}^{2 d}\right)$ or not.

It seems that the invariance properties (2) in Proposition 1.13 concerning the choice of weight function are not possible for weights that are not moderate. On the other hand, (1) and (4) in Proposition 1.13 hold true for certain weights outside $\mathscr{P}_{E}\left(\mathbf{R}^{2 d}\right)$. In fact, in [25], certain weight classes which contain $\mathscr{P}_{E}\left(\mathbf{R}^{2 d}\right)$ as well as weights of the form

$$
\omega(x, \xi)=e^{ \pm r\left(|x|^{\frac{1}{s}}+|\xi|^{\frac{1}{s}}\right)}, \quad x, \xi \in \mathbf{R}^{d},
$$

when $r>0$ and $s>\frac{1}{2}$ are introduced. For corresponding (broader) families of modulation spaces it is then proved that Proposition 1.13 (1) and (4) hold true (with some modifications).

1.5. Bargmann transform and spaces of analytic functions. The Bargmann transform $\mathfrak{V}_{d}$ is the homeomorphism from the spaces in (1.15) to respective spaces in (1.16), given by $T_{\mathcal{A}} \circ T_{\mathcal{H}}^{-1}$, where $T_{\mathcal{H}}$ and $T_{\mathcal{A}}$ are given by (1.14). For distributions in $\mathscr{S}^{\prime}\left(\mathbf{R}^{d}\right)$, this definition agrees with the original definition of the Bargmann transform, given in $[1,2]$, in view of $[1,2,26]$. by

In fact, if $f \in L^{p}\left(\mathbf{R}^{d}\right)$ for some $p \in[1, \infty]$, then $\mathfrak{V}_{d} f$ is the entire function given

$$
\left(\mathfrak{V}_{d} f\right)(z)=\pi^{-d / 4} \int_{\mathbf{R}^{d}} \exp \left(-\frac{1}{2}\left(\langle z, z\rangle+|y|^{2}\right)+2^{1 / 2}\langle z, y\rangle\right) f(y) d y, \quad z \in \mathbf{C}^{d},
$$

which can also be formulated as

or

$$
\left(\mathfrak{V}_{d} f\right)(z)=\int_{\mathbf{R}^{d}} \mathfrak{A}_{d}(z, y) f(y) d y, \quad z \in \mathbf{C}^{d}
$$

$$
\left(\mathfrak{V}_{d} f\right)(z)=\left\langle f, \mathfrak{A}_{d}(z, \cdot)\right\rangle, \quad z \in \mathbf{C}^{d}
$$


where the Bargmann kernel $\mathfrak{A}_{d}$ is given by

$$
\mathfrak{A}_{d}(z, y)=\pi^{-d / 4} \exp \left(-\frac{1}{2}\left(\langle z, z\rangle+|y|^{2}\right)+2^{1 / 2}\langle z, y\rangle\right), \quad z \in \mathbf{C}^{d}, y \in \mathbf{R}^{d} .
$$

(Cf. [1, 2].) Here

$$
\langle z, w\rangle=\sum_{j=1}^{d} z_{j} w_{j} \quad \text { and } \quad(z, w)=\langle z, \bar{w}\rangle
$$

when

$$
z=\left(z_{1}, \ldots, z_{d}\right) \in \mathbf{C}^{d} \text { and } w=\left(w_{1}, \ldots, w_{d}\right) \in \mathbf{C}^{d}
$$

and otherwise $\langle\cdot, \cdot\rangle$ denotes the duality between test function spaces and their corresponding duals which is clear form the context. We note that the right-hand side in (1.28) makes sense when $f \in \mathcal{S}_{1 / 2}^{\prime}\left(\mathbf{R}^{d}\right)$ and defines an element in $A\left(\mathbf{C}^{d}\right)$, since $y \mapsto \mathfrak{A}_{d}(z, y)$ can be interpreted as an element in $\mathcal{S}_{1 / 2}\left(\mathbf{R}^{d}\right)$ with values in $A\left(\mathbf{C}^{d}\right)$.

It was proved by Bargmann in [1] that $f \mapsto \mathfrak{V}_{d} f$ is a bijective and isometric map from $L^{2}\left(\mathbf{R}^{d}\right)$ to the Hilbert space $A^{2}\left(\mathbf{C}^{d}\right)$, the set of entire functions $F$ on $\mathbf{C}^{d}$ which fullfils

$$
\|F\|_{A^{2}} \equiv\left(\int_{\mathbf{C}^{d}}|F(z)|^{2} d \mu(z)\right)^{1 / 2}<\infty .
$$

Recall, $d \mu(z)=\pi^{-d} e^{-|z|^{2}} d \lambda(z)$, where $d \lambda(z)$ is the Lebesgue measure on $\mathbf{C}^{d}$, and the scalar product on $A^{2}\left(\mathbf{C}^{d}\right)$ is given by

$$
(F, G)_{A^{2}} \equiv \int_{\mathbf{C}^{d}} F(z) \overline{G(z)} d \mu(z), \quad F, G \in A^{2}\left(\mathbf{C}^{d}\right) .
$$

For future references we note that the latter scalar product induces the bilinear form

$$
(F, G) \mapsto\langle F, G\rangle_{A^{2}}=\langle F, G\rangle_{A^{2}\left(\mathbf{C}^{d}\right)} \equiv \int_{\mathbf{C}^{d}} F(z) G(z) d \mu(z)
$$

on $A^{2}\left(\mathbf{C}^{d}\right) \times \overline{A^{2}\left(\mathbf{C}^{d}\right)}$.

In [1] it was also proved that the orthonormal basis $\left\{h_{\alpha}\right\}_{\alpha \in \mathbf{N}^{d}}$ in $L^{2}\left(\mathbf{R}^{d}\right)$ of Hermite functions is mapped to the orthonormal basis $\left\{e_{\alpha}\right\}_{\alpha \in \mathbf{N}^{d}}$ in $A^{2}\left(\mathbf{C}^{d}\right)$ (cf. (1.13)). Furthermore, there is a convenient reproducing formula on $A^{2}\left(\mathbf{C}^{d}\right)$. In fact, let $\Pi_{A}$ be the operator from $L^{2}(d \mu)$ to $A\left(\mathbf{C}^{d}\right)$, given by

$$
\left(\Pi_{A} F\right)(z)=\int_{\mathbf{C}^{d}} F(w) e^{(z, w)} d \mu(w), \quad z \in \mathbf{C}^{d} .
$$

Then it is proved in [1] that $\Pi_{A}$ is an orthonormal projection from $L^{2}(d \mu)$ to $A^{2}\left(\mathbf{C}^{d}\right)$.

From now on we assume that $\phi$ in the definition of the short-time Fourier transform is given by

$$
\phi(x)=\pi^{-d / 4} e^{-|x|^{2} / 2}, \quad x \in \mathbf{R}^{d},
$$

if nothing else is stated. For such $\phi$, it follows by straight-forward computations that the relationship between the Bargmann transform and the short-time Fourier transform is given by

$$
\mathfrak{V}_{d}=U_{\mathfrak{V}} \circ V_{\phi}, \quad \text { and } \quad U_{\mathfrak{V}}^{-1} \circ \mathfrak{V}_{d}=V_{\phi},
$$

where $U_{\mathfrak{V}}$ is the linear, continuous and bijective operator on $\mathscr{D}^{\prime}\left(\mathbf{R}^{2 d}\right) \simeq \mathscr{D}^{\prime}\left(\mathbf{C}^{d}\right)$, given by

$$
\left(U_{\mathfrak{V}} F\right)(x+i \xi)=(2 \pi)^{d / 2} e^{\left(|x|^{2}+|\xi|^{2}\right) / 2} e^{-i\langle x, \xi\rangle} F\left(2^{1 / 2} x,-2^{1 / 2} \xi\right), \quad x, \xi \in \mathbf{R}^{d},
$$

cf. [25]. 
Definition 1.15. Let $E$ be an ordered basis for $\mathbf{R}^{2 d}, U_{\mathfrak{V}}$ be the operator in (1.35), $\boldsymbol{p} \in[1, \infty]^{2 d}, \phi(x)=\pi^{-\frac{d}{4}} e^{-\frac{1}{2} \cdot|x|^{2}}$ and let $\omega$ be a weight on $\mathbf{R}^{2 d}$.

(1) The space $B_{E,(\omega)}^{p}\left(\mathbf{C}^{d}\right)$ consists of all $F \in L_{\text {loc }}^{1}\left(\mathbf{C}^{d}\right)$ such that

$$
\|F\|_{B_{E,(\omega)}^{p}} \equiv\left\|\left(U_{\mathfrak{V}}^{-1} F\right) \cdot \omega\right\|_{L_{E}^{p}}
$$

is finite;

(2) The space $A_{E,(\omega)}^{p}\left(\mathbf{C}^{d}\right)$ consists of all $F \in A\left(\mathbf{C}^{d}\right) \bigcap B_{E,(\omega)}^{p}\left(\mathbf{C}^{d}\right)$ with topology inherited from $B_{E,(\omega)}^{p}\left(\mathbf{C}^{d}\right)$.

We note that the spaces in Definition 1.15 are normed spaces.

For conveneincy we set $\|F\|_{B_{E,(\omega)}^{p}}=\infty$, when $F \notin B_{E,(\omega)}^{p}\left(\mathbf{C}^{d}\right)$ is measurable, and $\|F\|_{A_{E,(\omega)}^{p}}=\infty$, when $F \in A\left(\mathbf{C}^{d}\right) \backslash B_{E,(\omega)}^{p}\left(\mathbf{C}^{d}\right)$.

Remark 1.16. In Definitions 1.11 and 1.15, important cases appear when $E$ is the standard basis for $\mathbf{R}^{2 d}$ and $p_{1}=\cdots=p_{d}=p \in[1, \infty]$ and $p_{d+1}=\cdots=p_{2 d}=q \in$ $[1, \infty]$. For such choices of $E$ and $\boldsymbol{p}$ we set $L^{p, q}=L_{E}^{p}$,

$$
M_{(\omega)}^{p, q}=M_{E,(\omega)}^{p}, \quad A_{(\omega)}^{p, q}=A_{E,(\omega)}^{p} \quad \text { and } \quad B_{(\omega)}^{p, q}=B_{E,(\omega)}^{p} .
$$

We also set

$$
M_{(\omega)}^{p}=M_{(\omega)}^{p, p}, \quad A_{(\omega)}^{p}=A_{(\omega)}^{p, p} \quad \text { and } \quad B_{(\omega)}^{p}=B_{(\omega)}^{p, p} .
$$

If in addition $\omega=1$, then we set

$$
M_{E,(\omega)}^{p}=M_{E}^{p}, \quad M_{(\omega)}^{p, q}=M^{p, q} \quad \text { and } \quad M_{(\omega)}^{p}=M^{p},
$$

and similarly for $A_{E,(\omega)}^{p}$ and $B_{E,(\omega)}^{p}$ spaces.

If instead $E=\left\{e_{d+1}, \ldots, e_{2 d}, e_{1}, \ldots, e_{d}\right\}$ where $e_{1}, \ldots, e_{2 d}$ is the standard basis for $\mathbf{R}^{2 d}$ and $p_{1}=\cdots=p_{d}=q \in[1, \infty]$ and $p_{d+1}=\cdots=p_{2 d}=p \in[1, \infty]$, then we set $L_{*}^{p, q}=L_{E}^{p}$,

$$
W_{(\omega)}^{p, q}=M_{E,(\omega)}^{p}, \quad A_{*,(\omega)}^{p, q}=A_{E,(\omega)}^{p} \quad \text { and } \quad B_{*,(\omega)}^{p, q}=B_{E,(\omega)}^{p} .
$$

We notice that the space $W_{(\omega)}^{p, q}$ in Remark 1.16 is an example of a (weighted) Wiener amalgam space (cf. $[9,10])$. For future references we observe that the $B_{(\omega)}^{p}$ norm is given by

$$
\begin{aligned}
& \|F\|_{B_{(\omega)}^{p}}=2^{d / p}(2 \pi)^{-d / 2}\left(\int_{\mathbf{C}^{d}}\left|e^{-|z|^{2} / 2} F(z) \omega\left(2^{1 / 2} \bar{z}\right)\right|^{p} d \lambda(z)\right)^{1 / p} \\
& =2^{d / p}(2 \pi)^{-d / 2}\left(\iint_{\mathbf{R}^{2 d}}\left|e^{-\left(|x|^{2}+|\xi|^{2}\right) / 2} F(x+i \xi) \omega\left(2^{1 / 2} x,-2^{1 / 2} \xi\right)\right|^{p} d x d \xi\right)^{1 / p}
\end{aligned}
$$

(with obvious modifications when $p=\infty$ ). Especially it follows that the norm and scalar product in $B_{(\omega)}^{2}\left(\mathbf{C}^{d}\right)$ take the forms

$$
\begin{aligned}
\|F\|_{B_{(\omega)}^{2}} & =\left(\int_{\mathbf{C}^{d}}\left|F(z) \omega\left(2^{1 / 2} \bar{z}\right)\right|^{2} d \mu(z)\right)^{1 / 2}, \quad F \in B_{(\omega)}^{2}\left(\mathbf{C}^{d}\right), \\
(F, G)_{B_{(\omega)}^{2}} & =\int_{\mathbf{C}^{d}} F(z) \overline{G(z)} \omega\left(2^{1 / 2} \bar{z}\right)^{2} d \mu(z), \quad F, G \in B_{(\omega)}^{2}\left(\mathbf{C}^{d}\right)
\end{aligned}
$$

(cf. (1.29) and (1.30)). 
By the definitions and (1.34) it follows that the Bargmann transform is an isometric injection from $M_{E,(\omega)}^{p}\left(\mathbf{R}^{d}\right)$ to $A_{E,(\omega)}^{p}\left(\mathbf{C}^{d}\right)$. In fact, we have the following refinement. We omit the proof since the result is a special case of Theorem 4.8 in [26].

Proposition 1.17. Let $E$ be an ordered basis for $\mathbf{R}^{2 d}, \boldsymbol{p} \in[1, \infty]^{2 d}$, and $\omega$ be a weight on $\mathbf{R}^{2 d}$. Then the Bargmann transform is an isometric bijection from $M_{E,(\omega)}^{p}\left(\mathbf{R}^{d}\right)$ to $A_{E,(\omega)}^{p}\left(\mathbf{C}^{d}\right)$.

Finally, the $S C B$ transform (i.e. the Semi Conjugated Bargmann transform), $\mathfrak{V}_{\Theta, d_{2}, d_{1}}$ is defined as $\Theta_{C, 1} \circ \mathfrak{V}_{d_{2}+d_{1}}$ (cf. Definition 1.7). We also set $\mathfrak{V}_{\Theta, d}=\mathfrak{V}_{\Theta, d, d}$. Evidently, all properties of the Bargmann transform carry over to analogous properties for the SCB transform. Assume that $E$ is a basis for $\mathbf{R}^{2 d_{2}} \times \mathbf{R}^{2 d_{1}}, \boldsymbol{p} \in[1, \infty]^{2 d_{2}+2 d_{1}}$, $p, q \in(0, \infty]$ and that $\omega$ is a weight on $\mathbf{R}^{2 d_{2}} \times \mathbf{R}^{2 d_{1}}$ Then $\widehat{A}_{E,(\omega)}^{p}\left(\mathbf{C}^{d_{2}+d_{1}}\right)$ is the image of $A_{E,(\omega)}^{p}\left(\mathbf{C}^{d_{2}+d_{1}}\right)$ under the map $\Theta_{C, 1}$ with the topology defined by the norm

$$
\|a\|_{\widehat{A}_{E,(\omega)}^{p}} \equiv\left\|\Theta_{C, 1} a\right\|_{A_{E,\left(\Theta_{C, 1} \omega\right)}^{p}}, \quad a \in \widehat{A}_{E,(\omega)}^{p}\left(\mathbf{C}^{d_{2}+d_{1}}\right)
$$

The spaces

$$
\widehat{A}_{(\omega)}^{p, q}\left(\mathbf{C}^{d_{2}} \times \mathbf{C}^{d_{1}}\right), \quad \widehat{A}_{(\omega)}^{p}\left(\mathbf{C}^{d_{2}} \times \mathbf{C}^{d_{1}}\right), \quad \widehat{A}^{p, q}\left(\mathbf{C}^{d_{2}} \times \mathbf{C}^{d_{1}}\right), \quad \widehat{A}^{p}\left(\mathbf{C}^{d_{2}} \times \mathbf{C}^{d_{1}}\right),
$$

their norms and the scalar product $(\cdot, \cdot)_{\widehat{A}^{2}}$ are defined analogously.

1.6. Pseudo-differential operators. Next we recall some properties in pseudodifferential calculus. Let $\mathbf{M}(d, \Omega)$ be the set of $d \times d$-matrices with entries in the set $\Omega, a \in \Sigma_{1}\left(\mathbf{R}^{2 d}\right)$, and let $A \in \mathbf{M}(d, \mathbf{R})$ be fixed. Then the pseudo-differential operator $\mathrm{Op}_{A}(a)$ is the linear and continuous operator on $\Sigma_{1}\left(\mathbf{R}^{d}\right)$, given by

$$
\left(\mathrm{Op}_{A}(a) f\right)(x)=(2 \pi)^{-d} \iint a(x-A(x-y), \xi) f(y) e^{i\langle x-y, \xi\rangle} d y d \xi, \quad x \in \mathbf{R}^{d} .
$$

For general $a \in \Sigma_{1}^{\prime}\left(\mathbf{R}^{2 d}\right)$, the pseudo-differential operator $\mathrm{Op}_{A}(a)$ is defined as the continuous operator from $\Sigma_{1}\left(\mathbf{R}^{d}\right)$ to $\Sigma_{1}^{\prime}\left(\mathbf{R}^{d}\right)$ with distribution kernel

$$
K_{a, A}(x, y)=(2 \pi)^{-d / 2}\left(\mathscr{F}_{2}^{-1} a\right)(x-A(x-y), x-y), \quad x, y \in \mathbf{R}^{d} .
$$

Here $\mathscr{F}_{2} F$ is the partial Fourier transform of $F(x, y) \in \Sigma_{1}^{\prime}\left(\mathbf{R}^{2 d}\right)$ with respect to the $y$ variable. This definition makes sense since the mappings

$$
\mathscr{F}_{2} \quad \text { and } \quad F(x, y) \mapsto F(x-A(x-y), x-y)
$$

are homeomorphisms on $\Sigma_{1}^{\prime}\left(\mathbf{R}^{2 d}\right)$. In particular, the map $a \mapsto K_{a, A}$ is a homeomorphism on $\Sigma_{1}^{\prime}\left(\mathbf{R}^{2 d}\right)$.

The standard (Kohn-Nirenberg) representation, $a(x, D)=\mathrm{Op}(a)$, and the Weyl quantization $\mathrm{Op}^{w}(a)$ of $a$ are obtained by choosing $A=0$ and $A=\frac{1}{2} I$, respectively, in (1.37) and (1.38), where $I=I_{d}$ is the $d \times d$ identity matrix.

Remark 1.18. By Fourier's inversion formula, (1.38) and the kernel theorem [16, Theorem 2.2], [22, Theorem 2.5] for operators from Gelfand-Shilov spaces to their duals, it follows that the map $a \mapsto \mathrm{Op}_{A}(a)$ is bijective from $\Sigma_{1}^{\prime}\left(\mathbf{R}^{2 d}\right)$ to the set of all linear and continuous operators from $\Sigma_{1}\left(\mathbf{R}^{d}\right)$ to $\Sigma_{1}^{\prime}\left(\mathbf{R}^{d}\right)$.

By Remark 1.18, it follows that for every $a_{1} \in \Sigma_{1}^{\prime}\left(\mathbf{R}^{2 d}\right)$ and $A_{1}, A_{2} \in \mathbf{M}(d, \mathbf{R})$, there is a unique $a_{2} \in \Sigma_{1}^{\prime}\left(\mathbf{R}^{2 d}\right)$ such that $\mathrm{Op}_{A_{1}}\left(a_{1}\right)=\mathrm{Op}_{A_{2}}\left(a_{2}\right)$. By Section $18.5 \mathrm{in}$ [15], the relation between $a_{1}$ and $a_{2}$ is given by

$$
\mathrm{Op}_{A_{1}}\left(a_{1}\right)=\mathrm{Op}_{A_{2}}\left(a_{2}\right) \Longleftrightarrow a_{2}=e^{i\left\langle\left(A_{1}-A_{2}\right) D_{\xi}, D_{x}\right\rangle} a_{1}
$$


Here we note that the operator $e^{i\left\langle A D_{\xi}, D_{x}\right\rangle}$ is homeomorphic on $\Sigma_{1}\left(\mathbf{R}^{2 d}\right)$ and its dual (cf. $[5,6,29]$ ). For modulation spaces we have the following subresult of Proposition 2.8 in [28]. Here and in what follows, $A^{*}$ is the transpose of $A \in \mathbf{M}(d, \mathbf{R})$.

Proposition 1.19. Let $s \geqslant \frac{1}{2}, A \in \mathbf{M}(d, \mathbf{R}), p, q \in(0, \infty], \phi, a \in \Sigma_{1}\left(\mathbf{R}^{2 d}\right)$ and let $T_{A}=e^{i\left\langle A D_{\xi}, D_{x}\right\rangle}$. If $\omega \in \mathscr{P}_{E}\left(\mathbf{R}^{4 d}\right)$ and

$$
\omega_{A}(x, \xi, \eta, y)=\omega\left(x+A y, \xi+A^{*} \eta, \eta, y\right),
$$

then $T_{A}$ from $\Sigma_{1}\left(\mathbf{R}^{2 d}\right)$ to $\Sigma_{1}\left(\mathbf{R}^{2 d}\right)$ extends uniquely to a homeomorphism from $M_{(\omega)}^{p, q}\left(\mathbf{R}^{2 d}\right)$ to $M_{\left(\omega_{A}\right)}^{p, q}\left(\mathbf{R}^{2 d}\right)$, and

$$
\left\|T_{A} a\right\|_{M_{\left(\omega_{A}\right)}^{p, q}}=\|a\|_{M_{(\omega)}^{p, q}}
$$

\section{Kernel theorems and analytic pseudo-differential operators}

In the first part of the section we show that there is a one to one correspondence between linear and continuous mappings from $\mathcal{A}_{s}^{\prime}$ to $\mathcal{A}_{s}\left(\mathcal{A}_{s}\right.$ to $\left.\mathcal{A}_{s}^{\prime}\right)$ and mappings with kernels in $\widehat{A}_{s}\left(\widehat{A}_{s}^{\prime}\right)$ with respect to the measure $d \mu$ (cf. Propositions 2.2 and 2.3). Thereafter we deduce in Theorems 2.7-2.10 analogous results for analytic pseudodifferential operators based on Theorem 2.6 which deals with mapping properties of the operator which takes $a(z, w)$ into $e^{(z, w)} a(z, w)$.

Here and in what follows, any extension of the $A^{2}$-form, $(\cdot, \cdot)_{A^{2}}$ from $\mathcal{A}_{0}\left(\mathbf{C}^{d}\right) \times$ $\mathcal{A}_{0}\left(\mathbf{C}^{d}\right)$ to $\mathbf{C}$ is still called $A^{2}$-form and still denoted by $(\cdot, \cdot)_{A^{2}}$. Similar approaches yield extensions of the forms $\langle\cdot, \cdot\rangle_{A^{2}}$ and $(\cdot, \cdot)_{\widehat{A}^{2}}$.

By the definitions, $\ell_{s}^{\prime}\left(\mathbf{N}^{d}\right)$ and $\ell_{0, s}^{\prime}\left(\mathbf{N}^{d}\right)$ are the duals of $\ell_{s}\left(\mathbf{N}^{d}\right)$ and $\ell_{0, s}\left(\mathbf{N}^{d}\right)$, respectively, through unique extensions of the $\ell^{2}\left(\mathbf{N}^{d}\right)$ form on $\ell_{0}\left(\mathbf{N}^{d}\right)$. Since the spaces in (1.16) are images of the spaces in (1.12) under the map $T_{\mathcal{A}}$ in (1.14), the following lemma is an immediate consequence of these duality properties. The result is also implicitly given in $[7,26]$.

Lemma 2.1. Let $s \in \overline{\mathbf{R}_{b}}$. Then the following is true:

(1) the form $(F, G) \mapsto(F, G)_{A^{2}}$ from $\mathcal{A}_{0}\left(\mathbf{C}^{d}\right) \times \mathcal{A}_{0}\left(\mathbf{C}^{d}\right)$ to $\mathbf{C}$ is uniquely extendable to continuous forms from $\mathcal{A}_{s}\left(\mathbf{C}^{d}\right) \times \mathcal{A}_{s}^{\prime}\left(\mathbf{C}^{d}\right)$ to $\mathbf{C}$, and from $\mathcal{A}_{0, s}\left(\mathbf{C}^{d}\right) \times \mathcal{A}_{0, s}^{\prime}\left(\mathbf{C}^{d}\right)$ to $\mathbf{C}$. Furthermore, the duals of $\mathcal{A}_{s}\left(\mathbf{C}^{d}\right)$ and $\mathcal{A}_{0, s}\left(\mathbf{C}^{d}\right)$ can be identified by $\mathcal{A}_{s}^{\prime}\left(\mathbf{C}^{d}\right)$ and $\mathcal{A}_{0, s}^{\prime}\left(\mathbf{C}^{d}\right)$ through the form $(\cdot, \cdot)_{A_{2}}$;

(2) the form $(F, G) \mapsto\langle F, G\rangle_{A^{2}}$ from $\mathcal{A}_{0}\left(\mathbf{C}^{d}\right) \times \overline{\mathcal{A}_{0}\left(\mathbf{C}^{d}\right)}$ to $\mathbf{C}$ is uniquely extendable to continuous forms from $\mathcal{A}_{s}\left(\mathbf{C}^{d}\right) \times \overline{\mathcal{A}_{s}^{\prime}\left(\mathbf{C}^{d}\right)}$ to $\mathbf{C}$, and from $\mathcal{A}_{0, s}\left(\mathbf{C}^{d}\right) \times \overline{\mathcal{A}_{0, s}^{\prime}\left(\mathbf{C}^{d}\right)}$ to $\mathbf{C}$. Furthermore, the duals of $\mathcal{A}_{s}\left(\mathbf{C}^{d}\right)$ and $\mathcal{A}_{0, s}\left(\mathbf{C}^{d}\right)$ can be identified by $\mathcal{A}_{s}^{\prime}\left(\mathbf{C}^{d}\right)$ and $\mathcal{A}_{0, s}^{\prime}\left(\mathbf{C}^{d}\right)$ through the form $\langle\cdot, \cdot\rangle_{A_{2}}$.

The following two propositions follow by applying $\mathfrak{V}_{\Theta, d_{2}, d_{1}}$ on Theorem 3.3 and 3.4 in [7], and using Lemma 2.1. The details are left for the reader.

Proposition 2.2. Let $s \in \overline{\mathbf{R}_{b}}$, and let $T$ be a linear and continuous map from $\mathcal{A}_{0}\left(\mathbf{C}^{d_{1}}\right)$ to $\mathcal{A}_{0}^{\prime}\left(\mathbf{C}^{d_{2}}\right)$. Then the following is true:

(1) if $T$ is a linear and continuous map from $\mathcal{A}_{s}^{\prime}\left(\mathbf{C}^{d_{1}}\right)$ to $\mathcal{A}_{s}\left(\mathbf{C}^{d_{2}}\right)$, then there is a unique $K \in \hat{\mathcal{A}}_{s}\left(\mathbf{C}^{d_{2}} \times \mathbf{C}^{d_{1}}\right)$ such that

$$
T F=\left(z_{2} \mapsto\left\langle K\left(z_{2}, \cdot\right), F\right\rangle_{A^{2}\left(\mathbf{C}^{d_{1}}\right)}\right)
$$

holds true;

(2) if $T$ is a linear and continuous map from $\mathcal{A}_{s}\left(\mathbf{C}^{d_{1}}\right)$ to $\mathcal{A}_{s}^{\prime}\left(\mathbf{C}^{d_{2}}\right)$, then there is a unique $K \in \widehat{\mathcal{A}}_{s}^{\prime}\left(\mathbf{C}^{d_{2}} \times \mathbf{C}^{d_{1}}\right)$ such that (2.1) holds true. 
The same holds true if $\mathcal{A}_{s}, \widehat{\mathcal{A}}_{s}, \mathcal{A}_{s}^{\prime}$ and $\widehat{\mathcal{A}}_{s}^{\prime}$ are replaced by $\mathcal{A}_{0, s}, \widehat{\mathcal{A}}_{0, s}, \mathcal{A}_{0, s}^{\prime}$ and $\widehat{\mathcal{A}}_{0, s}^{\prime}$, respectively, at each occurrence.

Proposition 2.3. Let $K \in \widehat{\mathcal{A}}_{0}^{\prime}\left(\mathbf{C}^{d_{2}} \times \mathbf{C}^{d_{1}}\right), s \in \overline{\mathbf{R}_{b}}$ and let $T$ be the linear and continuous map from $\mathcal{A}_{0}\left(\mathbf{C}^{d_{1}}\right)$ to $\mathcal{A}_{0}^{\prime}\left(\mathbf{C}^{d_{2}}\right)$, given by

$$
F \mapsto T F=\left(z_{2} \mapsto\left\langle K\left(z_{2}, \cdot\right), F\right\rangle_{A^{2}\left(\mathbf{C}^{d_{1}}\right)}\right)
$$

Then the following is true:

(1) if $K \in \widehat{\mathcal{A}}_{s}\left(\mathbf{C}^{d_{2}} \times \mathbf{C}^{d_{1}}\right)$, then $T$ extends uniquely to a linear and continuous map from $\mathcal{A}_{s}^{\prime}\left(\mathbf{C}^{d_{1}}\right)$ to $\mathcal{A}_{s}\left(\mathbf{C}^{d_{2}}\right)$;

(2) if $K \in \widehat{\mathcal{A}}_{s}^{\prime}\left(\mathbf{C}^{d_{2}} \times \mathbf{C}^{d_{1}}\right)$, then $T$ extends uniquely to a linear and continuous map from $\mathcal{A}_{s}\left(\mathbf{C}^{d_{1}}\right)$ to $\mathcal{A}_{s}^{\prime}\left(\mathbf{C}^{d_{2}}\right)$.

The same holds true if $\mathcal{A}_{s}, \hat{\mathcal{A}}_{s}, \mathcal{A}_{s}^{\prime}$ and $\hat{\mathcal{A}}_{s}^{\prime}$ are replaced by $\mathcal{A}_{0, s}, \hat{\mathcal{A}}_{0, s}, \mathcal{A}_{0, s}^{\prime}$ and $\hat{\mathcal{A}}_{0, s}^{\prime}$, respectively, at each occurrence.

The operator $T$ in (2.2) should be interpreted as $T$ in the formula

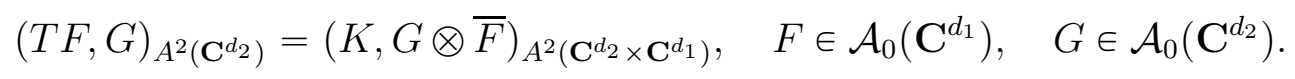

Next we recall the definition of analytic pseudo-differential operators. (See [25, Definition 6.20] in the case $t=0$, as well as [3, 4].)

Definition 2.4. Let $a \in \hat{\mathcal{A}}_{b_{1}}^{\prime}\left(\mathbf{C}^{d} \times \mathbf{C}^{d}\right)$. Then the analytic pseudo-differential operator $\mathrm{Op}_{\mathfrak{V}}(a)(\mathrm{A} \Psi \mathrm{DO})$ with symbol $a$ is given by

$$
\begin{aligned}
\left(\mathrm{Op}_{\mathfrak{V}}(a) F\right)(z) & =\int_{\mathbf{C}^{d}} a(z, w) F(w) e^{(z, w)} d \mu(w) \\
& =\left(F, \overline{a(z, \cdot)} e^{(\cdot, z)}\right)_{A^{2}\left(\mathbf{C}^{d}\right)}, \quad z \in \mathbf{C}^{d} .
\end{aligned}
$$

By the definition it follows that the relation between the operator kernel $K$ and the symbol $a$ is given by

$$
K(z, w)=e^{(z, w)} a(z, w), \quad z, w \in \mathbf{C}^{d},
$$

provided the multiplication on the right-hand side makes sense. This leads to the question about mapping properties of $T_{t}$ defined by

$$
\left(T_{t} a\right)(z, w)=e^{t(z, w)} a(z, w), \quad z, w \in \mathbf{C}^{d}, t \in \mathbf{C}
$$

when $a$ belongs to a suitable subspace of $\hat{\mathcal{A}}_{s}^{\prime}\left(\mathbf{C}^{2 d}\right)$.

First we notice that $a \in \widehat{A}\left(\mathbf{C}^{2 d}\right)$, if and only if $T_{t} a \in \widehat{A}\left(\mathbf{C}^{2 d}\right)$, and that the inverse of $T_{t}$ is $T_{-t}$. Hence $T_{t}$ is well-defined and a homeomorphism on $\widehat{\mathcal{A}}_{b_{1}}^{\prime}\left(\mathbf{C}^{2 d}\right)$.

If $T_{\mathcal{H}}$ is the same as in (1.14) then we shall investigate the map $T_{0, t}$ in the commutative diagram:

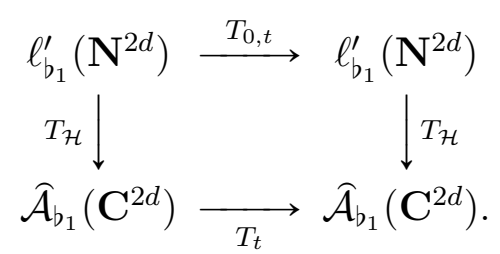

Therefore, let $a \in \widehat{\mathcal{A}}_{b_{1}}^{\prime}\left(\mathbf{C}^{2 d}\right)$ with the expansion

$$
a(z, w)=\sum_{\alpha, \beta \in \mathbf{N}^{d}} c(\alpha, \beta) e_{\alpha}(z) e_{\beta}(\bar{w})=\sum_{\alpha, \beta \in \mathbf{N}^{d}} c(\alpha, \beta) \frac{z^{\alpha} \bar{w}^{\beta}}{\sqrt{\alpha ! \beta !}}, \quad z, w \in \mathbf{C}^{d},
$$


where

$$
|c(\alpha, \beta)| \lesssim r^{|\alpha+\beta|} \sqrt{\alpha ! \beta !}
$$

for every $r>0$. Since

$$
e^{t(z, w)}=\sum_{\gamma \in \mathbf{N}^{d}} \frac{t^{|\gamma|} z^{\gamma} \bar{w}^{\gamma}}{\gamma !}, \quad z, w \in \mathbf{C}^{d}
$$

we have

$$
e^{t(z, w)} a(z, w)=\sum_{\gamma \in \mathbf{N}^{d}} \sum_{\alpha, \beta \in \mathbf{N}^{d}} \varphi_{t, z, w}(\alpha, \beta, \gamma), \quad z, w \in \mathbf{C}^{d}, t \in \mathbf{C}
$$

where

$$
\varphi_{t, z, w}(\alpha, \beta, \gamma)=\frac{c(\alpha, \beta) t^{|\gamma|} z^{\alpha+\gamma} \bar{w}^{\beta+\gamma}}{\gamma ! \sqrt{\alpha ! \beta !}}, \quad z, w \in \mathbf{C}^{d}, t \in \mathbf{C},
$$

We shall prove that the series in (2.7) is locally uniformly convergent with respect to $t, z$ and $w$. If $|t|<R,|z|<R$ and $|w|<R$ for some fixed $R>0$, then by (2.6) we get

$$
\left|\varphi_{t, z, w}(\alpha, \beta, \gamma)\right| \lesssim \frac{r^{|\alpha+\beta|} R^{|\alpha+\beta+2 \gamma|}}{\gamma !} \leqslant \frac{(r R)^{|\alpha+\beta|}\left(d R^{2}\right)^{|\gamma|}}{|\gamma| !}
$$

for all $\alpha, \beta, \gamma \in \mathbf{N}^{d}$. Since the series

$$
\sum_{\alpha, \beta, \gamma \in \mathbf{N}^{d}} \frac{(r R)^{|\alpha+\beta|}\left(2 R^{2}\right)^{|\gamma|}}{|\gamma| !}
$$

is convergent when $r$ is chosen strictly smaller than $R^{-1}$, the asserted uniform convergence follows from Weierstrass' theorem.

In particular, we may change the order of summation in (2.7) to obtain

$$
\begin{aligned}
\left(T_{t} a\right)(z, w) & =\sum_{\alpha, \beta \in \mathbf{N}^{d}} \sum_{\gamma \in \mathbf{N}^{d}} \frac{c(\alpha, \beta) t^{|\gamma|} z^{\alpha+\gamma} \bar{w}^{\beta+\gamma}}{\gamma ! \sqrt{\alpha ! \beta !}} \\
& =\sum_{\alpha, \beta \in \mathbf{N}^{d}} \sum_{\gamma \in \mathbf{N}^{d}} c(\alpha, \beta) t^{|\gamma|}\left(\left(\begin{array}{c}
\alpha+\gamma \\
\gamma
\end{array}\right)\left(\begin{array}{c}
\beta+\gamma \\
\gamma
\end{array}\right)\right)^{1 / 2} e_{\alpha+\gamma}(z) e_{\beta+\gamma}(\bar{w}) \\
& =\sum_{\alpha, \beta \in \mathbf{N}^{d}}\left(T_{0, t} c\right)(\alpha, \beta) e_{\alpha}(z) e_{\beta}(\bar{w}), \quad z, w \in \mathbf{C}^{d}, t \in \mathbf{C}
\end{aligned}
$$

where

$$
\left(T_{0, t} c\right)(\alpha, \beta)=\sum_{\gamma \leqslant \alpha, \beta} c(\alpha-\gamma, \beta-\gamma) t^{|\gamma|}\left(\left(\begin{array}{l}
\alpha \\
\gamma
\end{array}\right)\left(\begin{array}{l}
\beta \\
\gamma
\end{array}\right)\right)^{1 / 2}, \quad t \in \mathbf{C},
$$

and we have identified $T_{0, t}$ in the diagram (2.5).

We have now the following:

Proposition 2.5. Let $K \subseteq \mathbf{C}$ be compact, $t \in K, s, s_{0} \in \overline{\mathbf{R}_{b}}$ be such that $s<1 / 2$ and $0<s_{0} \leqslant 1 / 2$, and let $T_{0, t}$ be the map on $\ell_{0}^{\prime}\left(\mathbf{N}^{2 d}\right)$ given by (2.9). Then $T_{0, t}$ is a continuous and bijective map on $\ell_{0}^{\prime}\left(\mathbf{N}^{2 d}\right)$ with the inverse $T_{0,-t}$. Furthermore, $T_{0, t}$ restricts to homeomorphism from $\ell_{s}^{\prime}\left(\mathbf{N}^{2 d}\right)$ to $\ell_{s}^{\prime}\left(\mathbf{N}^{2 d}\right)$, and from $\ell_{0, s_{0}}^{\prime}\left(\mathbf{N}^{2 d}\right)$ to $\ell_{0, s_{0}}^{\prime}\left(\mathbf{N}^{2 d}\right)$. 
Proof. The topology on $\ell_{0}^{\prime}\left(\mathbf{N}^{2 d}\right)$ can be defined by the family of semi-norms

$$
p_{N}\left(\{c(\alpha, \beta)\}_{\alpha, \beta \in \mathbf{N}^{d}}\right)=\sup _{|\alpha| \leqslant N|\beta| \leqslant N} \sup _{|c(\alpha, \beta)|, \quad N \in \mathbf{N} .}
$$

Then, for a given $c \in \ell_{0}^{\prime}\left(\mathbf{N}^{2 d}\right)$ we have

$$
\begin{aligned}
p_{N}\left(T_{0, t}(c)\right) & \leqslant \sup _{|\alpha|,|\beta| \leqslant N} \sum_{\gamma \leqslant \alpha, \beta} c(\alpha-\gamma, \beta-\gamma)|t|^{|\gamma|}\left(\left(\begin{array}{l}
\alpha \\
\gamma
\end{array}\right)\left(\begin{array}{l}
\beta \\
\gamma
\end{array}\right)\right)^{1 / 2} \\
& \leqslant p_{N}(c) \sum_{|\gamma| \leqslant N}|t|^{|\gamma|} 2^{N} \leqslant 2^{N}(1+|t|)^{N} p_{N}(c), \quad t \in \mathbf{C}, \quad N \in \mathbf{N}
\end{aligned}
$$

and the continuity of $T_{0, t}$ on $\ell_{0}^{\prime}\left(\mathbf{N}^{2 d}\right)$ follows. By straight-forward computations it also follows that $T_{0,-t}$ is the inverse of $T_{0, t}$, which gives asserted homeomorphism properties of $T_{0, t}$ on $\ell_{0}^{\prime}\left(\mathbf{N}^{2 d}\right)$.

Next we consider the case when $s, s_{0} \in \mathbf{R}_{+}$. Assume that

$$
|c(\alpha, \beta)| \lesssim e^{\frac{1}{h}\left(|\alpha|^{1 / 2 s}+|\beta|^{1 / 2 s}\right)}, \quad \alpha, \beta \in \mathbf{N}^{d}
$$

for some constant $h>0$. Then

$$
\left(T_{0, t} c\right)(\alpha, \beta) \lesssim \sum_{\gamma \leqslant \alpha, \beta} e^{\frac{1}{h}\left(|\alpha-\gamma|^{1 / 2 s}+|\beta-\gamma|^{1 / 2 s}\right)}|t|^{|\gamma|}\left(\left(\begin{array}{l}
\alpha \\
\gamma
\end{array}\right)\left(\begin{array}{l}
\beta \\
\gamma
\end{array}\right)\right)^{1 / 2} \leqslant I(\alpha) I(\beta),
$$

where

$$
I(\alpha)=\left(\sum_{\gamma \leqslant \alpha}\left(\begin{array}{l}
\alpha \\
\gamma
\end{array}\right) e^{\frac{2}{h}\left(|\alpha-\gamma|^{1 / 2 s}\right)}|t|^{|\gamma|}\right)^{1 / 2}, \quad t \in \mathbf{C}, \alpha \in \mathbf{N}^{d},
$$

and similarly for $I(\beta)$. Since

$$
I(\alpha) \leqslant e^{\frac{1}{h}|\alpha|^{1 / 2 s}}\left(\sum_{\gamma \leqslant \alpha}\left(\begin{array}{c}
\alpha \\
\gamma
\end{array}\right)|t|^{|\gamma|}\right)^{1 / 2}=e^{\frac{1}{h}|\alpha|^{1 / 2 s}}(1+|t|)^{|\alpha| / 2}, \quad t \in \mathbf{C}, \alpha \in \mathbf{N}^{d}
$$

we get

$$
\left|\left(T_{0, t} c\right)(\alpha, \beta)\right| \lesssim e^{\frac{1}{h}\left(|\alpha|^{1 / 2 s}+|\beta|^{1 / 2 s}\right)}(1+|t|)^{(|\alpha|+|\beta|) / 2} \lesssim e^{\frac{2}{h}\left(|\alpha|^{1 / 2 s}+|\beta|^{1 / 2 s}\right)}
$$

$t \in K, \alpha, \beta \in \mathbf{N}^{d}$, where the last inequality follows from the fact that $s<1 / 2$. This gives the continuity assertions for $T_{0, t}$ in the case when $s, s_{0} \in \mathbf{R}_{+}$and $s, s_{0}<1 / 2$.

For $s_{0}=1 / 2$ we have

$$
\left|\left(T_{0, t} c\right)(\alpha, \beta)\right| \lesssim e^{\frac{1}{h}(|\alpha|+|\beta|)}(1+|t|)^{(|\alpha|+|\beta|) / 2}=e^{\frac{1}{h_{1}}(|\alpha|+|\beta|)}, \quad t \in K, \alpha, \beta \in \mathbf{N}^{d},
$$

for some other choice of $h_{1}>0$ which only depend on $|t|, d$ and $h$ and the continuity of $T_{0, t}$ on $\ell_{0, \frac{1}{2}}^{\prime}\left(\mathbf{N}^{2 d}\right)$ follows.

It remains to consider the case when $s=s_{0}=b_{\sigma}$ for some $\sigma>0$. Assume that

$$
|c(\alpha, \beta)| \leqslant C r^{|\alpha+\beta|}(\alpha ! \beta !)^{\frac{1}{2 \sigma}}, \quad \alpha, \beta \in \mathbf{N}^{d},
$$


for some constants $C, r>0$. Then

$$
\begin{aligned}
\left|\left(T_{0, t} c\right)(\alpha, \beta)\right| & \leqslant C \sum_{\gamma \leqslant \alpha, \beta} r^{|\alpha+\beta-2 \gamma|}((\alpha-\gamma) !(\beta-\gamma) !)^{\frac{1}{2 \sigma}}|t|^{|\gamma|}\left(\left(\begin{array}{c}
\alpha \\
\gamma
\end{array}\right)\left(\begin{array}{l}
\beta \\
\gamma
\end{array}\right)\right)^{1 / 2} \\
& \leqslant C_{r} \sum_{\gamma \leqslant \alpha, \beta} r^{|\alpha+\beta|}(\alpha ! \beta !)^{\frac{1}{2 \sigma}}|t|^{|\gamma|} 2^{|\alpha+\beta| / 2} \\
& \leqslant C_{r}(2 r)^{|\alpha+\beta|}(\alpha ! \beta !)^{\frac{1}{2 \sigma}} \sum_{|\gamma| \leqslant|\alpha+\beta|}|t|^{|\gamma|} \\
& \leqslant C_{r}(2 r(1+|t|))^{|\alpha+\beta|}(\alpha ! \beta !)^{\frac{1}{2 \sigma}}, \quad t \in \mathbf{C}, \alpha, \beta \in \mathbf{N}^{d}
\end{aligned}
$$

where $C_{r}>0$ only depends on $C$ and $r$. This shows that $T_{0, t}$ is continuous on $\ell_{b_{\sigma}}^{\prime}\left(\mathbf{N}^{2 d}\right)$ and on $\ell_{0, b_{\sigma}}^{\prime}\left(\mathbf{N}^{2 d}\right)$.

We have now the following:

Theorem 2.6. Let $t \in \mathbf{C}, s, s_{0} \in \overline{\mathbf{R}_{b}}$ be such that $s<\frac{1}{2}$ and $0<s_{0} \leqslant \frac{1}{2}$, and let $T_{t}$ be given by (2.4) when $a \in \widehat{\mathcal{A}}_{b_{1}}^{\prime}\left(\mathbf{C}^{2 d}\right)$. Then the following is true:

(1) $T_{t}$ restricts to a homeomorphism from $\hat{\mathcal{A}}_{0,1 / 2}^{\prime}\left(\mathbf{C}^{2 d}\right)$ to $\hat{\mathcal{A}}_{0,1 / 2}^{\prime}\left(\mathbf{C}^{2 d}\right)$;

(2) $T_{t}$ from $\hat{\mathcal{A}}_{0,1 / 2}^{\prime}\left(\mathbf{C}^{2 d}\right)$ to $\hat{\mathcal{A}}_{0,1 / 2}^{\prime}\left(\mathbf{C}^{2 d}\right)$ extends uniquely to homeomorphisms from $\hat{\mathcal{A}}_{s}^{\prime}\left(\mathbf{C}^{2 d}\right)$ to $\widehat{\mathcal{A}}_{s}^{\prime}\left(\mathbf{C}^{2 d}\right)$ and from $\widehat{\mathcal{A}}_{0, s_{0}}^{\prime}\left(\mathbf{C}^{2 d}\right)$ to $\widehat{\mathcal{A}}_{0, s_{0}}^{\prime}\left(\mathbf{C}^{2 d}\right)$.

Proof. By the commutative diagram (2.5) we have

$$
T_{t} a=\left(T_{1} \circ T_{0, t} \circ T_{1}^{-1}\right) a, \quad a \in \widehat{\mathcal{A}}_{b_{1}}\left(\mathbf{C}^{2 d}\right),
$$

and letting $T_{t} a=\left(T_{1} \circ T_{0, t} \circ T_{1}^{-1}\right) a$ for general $a \in \widehat{\mathcal{A}}_{0}^{\prime}\left(\mathbf{C}^{2 d}\right)$, the continuity assertions follow from Proposition 2.5.

It remains to prove the uniqueness. Let $a \in \widehat{\mathcal{A}}_{0}^{\prime}\left(\mathbf{C}^{2 d}\right), b \in \widehat{\mathcal{A}}_{0}\left(\mathbf{C}^{2 d}\right)$, with the corresponding expansion coefficients $c_{a}(\alpha, \beta)$, and $c_{b}(\alpha, \beta)$, respectively, and let $c_{T_{t} a}(\alpha, \beta)$ be the coefficients of $T_{t} a \in \widehat{\mathcal{A}}_{0}^{\prime}\left(\mathbf{C}^{2 d}\right), \alpha, \beta \in \mathbf{N}^{d}$. Then

$$
(a, b)_{\widehat{A}^{2}}=\sum_{|\alpha+\beta| \leqslant N} c_{a}(\alpha, \beta) \overline{c_{b}(\alpha, \beta)}
$$

for some $N \in \mathbf{N}$ depending on $b$. Now choose a sequence $a_{j} \in \widehat{\mathcal{A}}_{0}\left(\mathbf{C}^{2 d}\right)$ such that

$$
\lim _{j \rightarrow \infty}\left(a_{j}, b\right)_{\widehat{A}^{2}}=(a, b)_{\widehat{A}^{2}}, \quad \text { for every } \quad b \in \widehat{\mathcal{A}}_{0}\left(\mathbf{C}^{2 d}\right) .
$$

If $c_{a_{j}}(\alpha, \beta)$ denote the coefficients in the expansion of $a_{j}, j \in \mathbf{N}$, then it follows from (2.10) that

$$
\lim _{j \rightarrow \infty} c_{a_{j}}(\alpha, \beta)=c_{a}(\alpha, \beta), \quad \text { for every } \quad \alpha, \beta \in \mathbf{N}^{d}
$$

by taking $b(z, w)=e_{\alpha}(z) e_{\beta}(\bar{w})$. The uniqueness follows if we prove that

$$
\lim _{j \rightarrow \infty}\left(\left(T_{t} a_{j}\right), b\right)_{\widehat{A}^{2}}=\left(\left(T_{t} a\right), b\right)_{\widehat{A}^{2}}, \quad \text { for every } \quad b \in \widehat{\mathcal{A}}_{0}\left(\mathbf{C}^{2 d}\right) .
$$

Let the coefficients of $T_{t} a_{j}$ be denoted by $c_{t, a_{j}}(\alpha, \beta), \alpha, \beta \in \mathbf{N}^{d}$. By (2.9) and (2.11) we get $c_{t, a_{j}}(\alpha, \beta) \rightarrow c_{t, a}(\alpha, \beta)$ as $j \rightarrow \infty$, for every $(\alpha, \beta) \in \mathbf{N}^{2 d}$, and $(2.12)$ follows since

$$
\left(\left(T_{t} a_{j}\right), b\right)_{\widehat{A}^{2}}=\sum_{|\alpha+\beta| \leqslant N} c_{t, a_{j}}(\alpha, \beta) \overline{c_{b}(\alpha, \beta)}
$$


and

$$
\left(\left(T_{t} a\right), b\right)_{\widehat{A}^{2}}=\sum_{|\alpha+\beta| \leqslant N} c_{t, a}(\alpha, \beta) \overline{c_{b}(\alpha, \beta)}, \quad b \in \widehat{\mathcal{A}}_{0}\left(\mathbf{C}^{2 d}\right)
$$

where $N$ depends on $b$ only.

The following two theorems now follows by combining Propositions 2.2 and 2.3 with Theorem 2.6. The details are left for the reader.

Theorem 2.7. Let $s \in \overline{\mathbf{R}_{b}}$ be such that $s<\frac{1}{2}$ and let $T$ be a linear and continuous map from $\mathcal{A}_{s}\left(\mathbf{C}^{d}\right)$ to $\mathcal{A}_{s}^{\prime}\left(\mathbf{C}^{d}\right)$. Then there is a unique $a \in \hat{\mathcal{A}}_{s}^{\prime}\left(\mathbf{C}^{d} \times \mathbf{C}^{d}\right)$ such that $T=\mathrm{Op}_{\mathfrak{V}}(a)$. The same holds true if $s<\frac{1}{2}, \mathcal{A}_{s}, \mathcal{A}_{s}^{\prime}$ and $\widehat{\mathcal{A}}_{s}^{\prime}$ are replaced by $s \leqslant \frac{1}{2}$, $\mathcal{A}_{0, s}, \mathcal{A}_{0, s}^{\prime}$ and $\widehat{\mathcal{A}}_{0, s}^{\prime}$, respectively, at each occurrence.

Theorem 2.8. Let $a \in \widehat{\mathcal{A}}_{0}^{\prime}\left(\mathbf{C}^{d} \times \mathbf{C}^{d}\right)$ and $s \in \overline{\mathbf{R}_{b}}$ be such that $s<\frac{1}{2}$. If $a \in \hat{\mathcal{A}}_{s}^{\prime}\left(\mathbf{C}^{d} \times \mathbf{C}^{d}\right)$, then $\mathrm{Op}_{\mathfrak{V}}(a)$ extends uniquely to a linear and continuous map from $\mathcal{A}_{s}\left(\mathbf{C}^{d}\right)$ to $\mathcal{A}_{s}^{\prime}\left(\mathbf{C}^{d}\right)$. The same holds true if $s<\frac{1}{2}, \mathcal{A}_{s}, \mathcal{A}_{s}^{\prime}$ and $\widehat{\mathcal{A}}_{s}^{\prime}$ are replaced by $s \leqslant \frac{1}{2}, \mathcal{A}_{0, s}, \mathcal{A}_{0, s}^{\prime}$ and $\hat{\mathcal{A}}_{0, s}^{\prime}$, respectively, at each occurrence.

The analogous results to Theorems 2.7 and 2.8 for larger $s$ are equivalent to kernel theorems for Fourier invariant Gelfand-Shilov spaces.

Theorem 2.9. Let $s \geqslant \frac{1}{2}\left(s>\frac{1}{2}\right)$. Then the following is true:

(1) If $T$ is a linear and continuous map from $\mathcal{A}_{s}^{\prime}\left(\mathbf{C}^{d}\right)$ to $\mathcal{A}_{s}\left(\mathbf{C}^{d}\right)$ (from $\mathcal{A}_{0, s}^{\prime}\left(\mathbf{C}^{d}\right)$ to $\left.\mathcal{A}_{0, s}\left(\mathbf{C}^{d}\right)\right)$, then there is a unique $a \in \widehat{A}\left(\mathbf{C}^{d} \times \mathbf{C}^{d}\right)$ such that

$$
|a(z, w)| \lesssim e^{\frac{1}{2} \cdot|z-w|^{2}-r\left(|z|^{\frac{1}{s}}+|w|^{\frac{1}{s}}\right)}, \quad z, w \in \mathbf{C}^{d},
$$

for some (for every) $r>0$ and $T=\mathrm{Op}_{\mathfrak{V}}(a)$;

(2) If $T$ is a linear and continuous map from $\mathcal{A}_{s}\left(\mathbf{C}^{d}\right)$ to $\mathcal{A}_{s}^{\prime}\left(\mathbf{C}^{d}\right)\left(\right.$ from $\mathcal{A}_{0, s}\left(\mathbf{C}^{d}\right)$ to $\left.\mathcal{A}_{0, s}^{\prime}\left(\mathbf{C}^{d}\right)\right)$, then there is a unique $a \in \widehat{A}\left(\mathbf{C}^{d} \times \mathbf{C}^{d}\right)$ such that

$$
|a(z, w)| \lesssim e^{\frac{1}{2} \cdot|z-w|^{2}+r\left(|z|^{\frac{1}{s}}+|w|^{\frac{1}{s}}\right)}, \quad z, w \in \mathbf{C}^{d},
$$

for every (for some) $r>0$ and $T=\mathrm{Op}_{\mathfrak{V}}(a)$.

Theorem 2.10. Let $s \geqslant \frac{1}{2}\left(s>\frac{1}{2}\right)$. Then the following is true:

(1) If $a \in \widehat{A}\left(\mathbf{C}^{d} \times \mathbf{C}^{d}\right)$ satisfies (2.13) for some (for every) $r>0$, then $\mathrm{Op}_{\mathfrak{V}}(a)$ from $\mathcal{A}_{0}\left(\mathbf{C}^{d}\right)$ to $\mathcal{A}_{0}^{\prime}\left(\mathbf{C}^{d}\right)$ is uniquely extendable to a linear and continuous map from $\mathcal{A}_{s}^{\prime}\left(\mathbf{C}^{d}\right)$ to $\mathcal{A}_{s}\left(\mathbf{C}^{d}\right)$ (from $\mathcal{A}_{0, s}^{\prime}\left(\mathbf{C}^{d}\right)$ to $\mathcal{A}_{0, s}\left(\mathbf{C}^{d}\right)$ );

(2) If $a \in \widehat{A}\left(\mathbf{C}^{d} \times \mathbf{C}^{d}\right)$ satisfies (2.14) for every (for some) $r>0$, then $\mathrm{Op}_{\mathfrak{V} \mathfrak{V}}(a)$ from $\mathcal{A}_{0}\left(\mathbf{C}^{d}\right)$ to $\mathcal{A}_{0}^{\prime}\left(\mathbf{C}^{d}\right)$ is uniquely extendable to a linear and continuous map from $\mathcal{A}_{s}\left(\mathbf{C}^{d}\right)$ to $\mathcal{A}_{s}^{\prime}\left(\mathbf{C}^{d}\right)$ (from $\mathcal{A}_{0, s}\left(\mathbf{C}^{d}\right)$ to $\mathcal{A}_{0, s}^{\prime}\left(\mathbf{C}^{d}\right)$ ).

Proof. We only prove the results for mappings between $\mathcal{A}_{s}$ and $\mathcal{A}_{s}^{\prime}$ spaces. The case when $\mathcal{A}_{0, s}$ and $\mathcal{A}_{0, s}^{\prime}$ spaces are involved follows by similar arguments and is left for the reader.

If $T$ is the same as in (2.2) for some $K \in \widehat{A}\left(\mathbf{C}^{d} \times \mathbf{C}^{d}\right)$, then $T=\mathrm{Op}_{\mathfrak{V}}(a)$ when $a(z, w)=e^{-(z, w)} K(z, w),(z, w) \in \mathbf{C}^{d} \times \mathbf{C}^{d}$. Since

$$
\left|e^{-(z, w)}\right| e^{\frac{1}{2}\left(|z|^{2}+|w|^{2}\right)}=e^{\frac{1}{2} \cdot|z-w|^{2}}, \quad z, w \in \mathbf{C}^{d},
$$


Theorem 1.9 gives

$$
\begin{aligned}
K \in \widehat{\mathcal{A}}_{s}\left(\mathbf{C}^{d} \times \mathbf{C}^{d}\right) & \Longleftrightarrow|K(z, w)| \lesssim e^{\frac{1}{2} \cdot\left(|z|^{2}+|w|^{2}\right)-r\left(|z|^{\frac{1}{s}}+|w|^{\frac{1}{s}}\right)} \\
& \Longleftrightarrow|a(z, w)| \lesssim e^{\frac{1}{2} \cdot\left(|z-w|^{2}\right)-r\left(|z|^{\frac{1}{s}}+|w|^{\frac{1}{s}}\right)}, \quad z, w \in \mathbf{C}^{d} .
\end{aligned}
$$

for some $r>0$. In the same way,

$$
K \in \widehat{\mathcal{A}}_{s}^{\prime}\left(\mathbf{C}^{d} \times \mathbf{C}^{d}\right) \Longleftrightarrow|a(z, w)| \lesssim e^{\frac{1}{2} \cdot\left(|z-w|^{2}\right)+r\left(|z|^{\frac{1}{s}}+|w|^{\frac{1}{s}}\right)}, \quad z, w \in \mathbf{C}^{d},
$$

for every $r>0$. The results now follows from these relations and Propositions 2.2 and 2.3

Remark 2.11. For strict subspaces of $\widehat{\mathcal{A}}_{0,1 / 2}^{\prime}\left(\mathbf{C}^{d}\right)$ in Definition 1.7, the estimates imposed on their elements are given by (1.23) or by (1.24) for suitable assumptions on $r>0$. It is evident that in all such cases, these conditions are violated under the action of $T_{t}$ in Theorem 2.6 when $t \neq 0$. Hence, Theorem 2.6 cannot be extended to other spaces in Definition 1.7.

In particular, the conditions (2.13) and (2.14) in Theorems 2.9 and 2.10 can not be replaced by the convenient condition that $a$ should belong to e.g.

$$
\widehat{\mathcal{A}}_{0, s}\left(\mathbf{C}^{d} \times \mathbf{C}^{d}\right), \quad \widehat{\mathcal{A}}_{s}\left(\mathbf{C}^{d} \times \mathbf{C}^{d}\right), \quad \widehat{\mathcal{A}}_{s_{1}}^{\prime}\left(\mathbf{C}^{d} \times \mathbf{C}^{d}\right) \quad \text { or } \quad \widehat{\mathcal{A}}_{0, s_{2}}^{\prime}\left(\mathbf{C}^{d} \times \mathbf{C}^{d}\right)
$$

when $s \in \overline{\mathbf{R}_{b}}, s_{1} \geqslant \frac{1}{2}$ and $s_{2}>\frac{1}{2}$. On the other hand, the conditions on $a$ in Theorems 2.9 and 2.10 mean exactly that $(z, w) \mapsto e^{(z, w)} a(z, w)$ belongs to the spaces in (2.15), depending on the choice between (2.13) and (2.14), and the condition on $r$.

Remark 2.12. Let $s \in \mathbf{R}_{b}$ be such that $s \leqslant \frac{1}{2}$. By similar arguments as in the proofs of Theorems 2.9 and 2.10, one may also characterize linear and continuous operators from $\mathcal{A}_{s}^{\prime}\left(\mathbf{C}^{d}\right)$ to $\mathcal{A}_{s}\left(\mathbf{C}^{d}\right)$, and from $\mathcal{A}_{0, s}^{\prime}\left(\mathbf{C}^{d}\right)$ to $\mathcal{A}_{0, s}\left(\mathbf{C}^{d}\right)$ as operators of the form $\mathrm{Op}_{\mathfrak{V}}(a)$ for suitable conditions on $a$. The details are left for the reader.

\section{Operators with kernels and symbols in mixed weighted Lebesgue spaces}

In this section we focus on operators in the previous section, whose kernels should belong to $\widehat{A}\left(\mathbf{C}^{d} \times \mathbf{C}^{d}\right)$ and obey certain mixed norm estimates of Lebesgue types. We deduce continuity properties of such operators when acting between suitable Lebesgue spaces of analytic functions. (See Theorems 3.3-3.5.) Thereafter we show that our results can be used to regain well-known and sharp continuity results in [27] for pseudo-differential operators with symbols in modulation spaces when acting on other modulation spaces. (See Theorems 3.8 and 3.9.) A key step here is to deduce an explicit formula which relates the short-time Fourier transform of the symbol to a real pseudo-differential operator $\operatorname{Op}(a)$ with the Bargmann transform of the kernel to $\operatorname{Op}(a)$. (See Lemma 3.7.)

We shall consider Lebesgue norm conditions of matrix pull-backs of the involved kernels. Let

$$
\begin{gathered}
C_{j k}^{l} \in \mathbf{M}(d, \mathbf{R}), \quad C_{j k}=\left(\begin{array}{ll}
C_{j, k}^{1} & C_{j, k}^{2} \\
C_{j, k}^{3} & C_{j, k}^{4}
\end{array}\right) \in \mathbf{M}(2 d, \mathbf{R}), \\
C=\left(\begin{array}{ll}
C_{11} & C_{12} \\
C_{21} & C_{22}
\end{array}\right) \in \mathbf{M}(4 d, \mathbf{R}), \quad j, k, l \in \mathbf{Z}_{+},
\end{gathered}
$$


and let

$$
\begin{aligned}
U_{d}(x+i \xi) & =(x, \xi) \in \mathbf{R}^{2 d}, \\
U_{d, d}\left(x_{1}+i \xi_{1}, x_{2}+i \xi_{2}\right) & =\left(x_{1}, \xi_{1}, x_{2}, \xi_{2}\right) \in \mathbf{R}^{4 d}, \\
K_{\omega}(z, w) & \equiv e^{-\frac{1}{2}\left(|z|^{2}+|w|^{2}\right)}|K(z, w)| \cdot \omega(\sqrt{2} \bar{z}, \sqrt{2} w), \\
G_{K, C, \omega} & =K_{\omega} \circ U_{d, d}^{-1} \circ C \circ U_{d, d}, \\
x, x_{j}, \xi, \xi_{j} & \in \mathbf{R}^{d}, \quad z, w \in \mathbf{C}^{d}, j=1,2 .
\end{aligned}
$$

We will consider continuity of operators from $A_{E,\left(\omega_{1}\right)}^{\boldsymbol{p}_{1}}\left(\mathbf{C}^{d}\right)$ to $A_{E,\left(\omega_{2}\right)}^{\boldsymbol{p}_{2}}\left(\mathbf{C}^{d}\right)$, when $G_{K, C, \omega}$ fullfils suitable $L^{p, q}\left(\mathbf{C}^{2 d}\right)$ estimates, where the weights fullfil

$$
\frac{\omega_{2}(z)}{\omega_{1}(w)} \lesssim \omega(z, \bar{w}), \quad z, w \in \mathbf{C}^{d}
$$

Here and in what follows we let $L^{p, q}\left(\mathbf{C}^{2 d}\right)$ and $L_{*}^{p, q}\left(\mathbf{C}^{2 d}\right)$ be the sets of all $G \in L_{\text {loc }}^{1}\left(\mathbf{C}^{2 d}\right)$ such that

$$
\|G\|_{L^{p, q}\left(\mathbf{C}^{2 d}\right)} \equiv\left\|G \circ U_{d, d}\right\|_{L^{p, q}\left(\mathbf{R}^{4 d}\right)} \quad \text { and } \quad\|G\|_{L_{*}^{p, q}\left(\mathbf{C}^{2 d}\right)} \equiv\left\|G \circ U_{d, d}\right\|_{L_{*}^{p, q}\left(\mathbf{R}^{4 d}\right)},
$$

respectively, are finite. (See also Remark 1.16.)

The involved Lebesgue exponents should satisfy

$$
\frac{1}{\boldsymbol{p}_{1}}-\frac{1}{\boldsymbol{p}_{2}}=1-\frac{1}{p}-\frac{1}{q}, \quad q \leqslant \boldsymbol{p}_{2} \leqslant p, \quad p, q \in[1, \infty], \quad \boldsymbol{p}_{1}, \boldsymbol{p}_{2} \in[1, \infty]^{2 d} .
$$

We need that $C$ and $C_{j k}$ above should satisfy

$$
\operatorname{det}(C) \operatorname{det}\left(C_{11} C_{21}\right) \neq 0
$$

or

$$
\operatorname{det}(C) \operatorname{det}\left(C_{12} C_{22}\right) \neq 0 .
$$

In (3.4) and in what follows we use the convention

$$
\frac{1}{\boldsymbol{p}}=\left(\frac{1}{p_{1}}, \ldots, \frac{1}{p_{d}}\right), \quad p_{0} \leqslant \boldsymbol{p}, \quad \boldsymbol{p} \leqslant p, \quad q_{0}<\boldsymbol{q}, \quad \boldsymbol{q}<q \quad \text { and } \quad \boldsymbol{r}=r,
$$

when

$$
\boldsymbol{p}=\left(p_{1}, \ldots, p_{d}\right), \quad \boldsymbol{q}=\left(q_{1}, \ldots, q_{d}\right), \quad \boldsymbol{r}=\left(r_{1}, \ldots, r_{d}\right)
$$

belong to $[1, \infty]^{d}$ and $p, q, r, p_{0}, q_{0}, r_{0} \in[1, \infty]$ satisfy

$$
p_{0} \leqslant p_{k}, \quad p_{k} \leqslant p, \quad q_{0}<q_{k}, \quad q_{k}<q \quad \text { and } \quad r_{k}=r, \quad k \in\{1, \ldots, d\} .
$$

Remark 3.1. We notice that (3.1)-(3.6) implies that $C$ is invertible and that at least one of the following conditions hold true:

(1) both $C_{11}$ and $C_{21}$ are invertible;

(2) both $C_{12}$ and $C_{22}$ are invertible.

If (1) holds, then

$$
\begin{aligned}
\operatorname{det}\left(\begin{array}{ll}
C_{11} & C_{12} \\
C_{21} & C_{22}
\end{array}\right) & =\operatorname{det}\left(\begin{array}{ll}
I_{2 d} & C_{11}^{-1} C_{12} \\
I_{2 d} & C_{21}^{-1} C_{22}
\end{array}\right)=\operatorname{det}\left(\begin{array}{cc}
I_{2 d} & C_{11}^{-1} C_{12} \\
0 & C_{21}^{-1} C_{22}-C_{11}^{-1} C_{12}
\end{array}\right) \\
& =\operatorname{det}\left(C_{21}^{-1} C_{22}-C_{11}^{-1} C_{12}\right) .
\end{aligned}
$$


Here recall that $I=I_{d}$ is the $d \times d$ identity matrix. From these computations it follows that

$$
\begin{array}{ll}
C_{11}^{-1} C_{12}-C_{21}^{-1} C_{22}, & C_{11}^{-1} C_{12}-C_{22} C_{21}^{-1}, \\
C_{12} C_{11}^{-1}-C_{21}^{-1} C_{22}, & C_{12} C_{11}^{-1}-C_{22} C_{21}^{-1}
\end{array}
$$

are invertible when (1) holds, and

$$
\begin{array}{ll}
C_{12}^{-1} C_{11}-C_{22}^{-1} C_{21}, & C_{12}^{-1} C_{11}-C_{21} C_{22}^{-1}, \\
C_{11} C_{12}^{-1}-C_{22}^{-1} C_{21}, & C_{11} C_{12}^{-1}-C_{21} C_{22}^{-1}
\end{array}
$$

are invertible when (2) holds.

Remark 3.2. Let $C_{0} \in \mathbf{M}(d, \mathbf{C})$ and let $U_{d}$ be the same as in (3.2). Then the matrix $U_{d} \circ C_{0} \circ U_{d}^{-1}$ which corresponds to $C_{0}$ is given by

$$
\left(\begin{array}{lr}
\operatorname{Re}\left(C_{0}\right) & -\operatorname{Im}\left(C_{0}\right) \\
\operatorname{Im}\left(C_{0}\right) & \operatorname{Re}\left(C_{0}\right)
\end{array}\right)
$$

Obviously, the map which takes $C_{0} \in \mathbf{M}(d, \mathbf{C})$ into the matrix (3.7) in $\mathbf{M}(2 d, \mathbf{R})$ is injective, but not bijective. In this way we identify $\mathbf{M}(d, \mathbf{C})$ with the set of all matrices in $\mathbf{M}(2 d, \mathbf{R})$ which are given by (3.7) for some $C_{0} \in \mathbf{M}(d, \mathbf{C})$.

If $C_{j k} \in \mathbf{M}(2 d, \mathbf{R})$ and $T_{j k}=U_{d}^{-1} \circ C_{j k} \circ U_{d}$, then $G_{K, C, \omega}$ in (3.2) is given by

$$
G_{K, C, \omega}(z, w)=K_{\omega}\left(T_{11}(z)+T_{12}(w), T_{21}(z)+T_{22}(w)\right), \quad z, w \in \mathbf{C}^{d} .
$$

If more restricted, $C_{j k}$ can be identified as matrices in $\mathbf{M}(d, \mathbf{C})$ as above, for $j, k \in\{1,2\}$, then $G_{K, C, \omega}$ in (3.2) is given by

$$
G_{K, C, \omega}(z, w)=K_{\omega}\left(C_{11} z+C_{12} w, C_{21} z+C_{22} w\right), \quad z, w \in \mathbf{C}^{d},
$$

for such choices of $C$.

Theorem 3.3. Let $E$ be an ordered basis for $\mathbf{R}^{2 d}, \omega_{1}$ and $\omega_{2}$ be weights on $\mathbf{C}^{d}$, $\omega$ be a weight on $\mathbf{C}^{d} \times \mathbf{C}^{d}$ such that (3.3) holds, and let $\boldsymbol{p}_{1}, \boldsymbol{p}_{2}, p$ and $q$ be as in (3.4). Also let $C \in \mathbf{M}(4 d, \mathbf{R})$ be such that (3.1) holds, $K \in \widehat{A}\left(\mathbf{C}^{d} \times \mathbf{C}^{d}\right)$, and let $G_{K, C, \omega}$ be as in (3.2). Then the following is true:

(1) if (3.5) holds and $G_{K, C, \omega}(z, w) \in L^{p, q}\left(\mathbf{C}^{2 d}\right)$, then $T_{K}$ in (2.1) from $\mathcal{A}_{b_{1}}\left(\mathbf{C}^{d}\right)$ to $A\left(\mathbf{C}^{d}\right)$ is uniquely extendable to a continuous mapping from $A_{E,\left(\omega_{1}\right)}^{\boldsymbol{p}_{1}}\left(\mathbf{C}^{d}\right)$ to $A_{E,\left(\omega_{2}\right)}^{p_{2}}\left(\mathbf{C}^{d}\right)$, and

$$
\left\|T_{K} F\right\|_{A_{E,\left(\omega_{2}\right)}^{p_{2}}} \lesssim\left\|G_{K, C, \omega}\right\|_{L^{p, q}}\|F\|_{A_{E,\left(\omega_{1}\right)}^{p_{1}}}, \quad F \in A_{E,\left(\omega_{1}\right)}^{p_{1}}\left(\mathbf{C}^{d}\right) ;
$$

(2) if (3.6) holds and $G_{K, C, \omega} \in L_{*}^{q, p}\left(\mathbf{C}^{2 d}\right)$, then $T_{K}$ in (2.1) from $\mathcal{A}_{b_{1}}\left(\mathbf{C}^{d}\right)$ to $A\left(\mathbf{C}^{d}\right)$ is uniquely extendable to a continuous mapping from $A_{E,\left(\omega_{1}\right)}^{p_{1}}\left(\mathbf{C}^{d}\right)$ to $A_{E,\left(\omega_{2}\right)}^{p_{2}}\left(\mathbf{C}^{d}\right)$, and

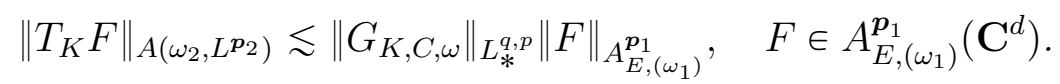

Proof. We only prove (1). The assertion (2) follows by similar arguments and is left for the reader. Let

$$
G_{K, C, \omega, p}(w) \equiv\left\|G_{K, C, \omega}(\cdot, w)\right\|_{L^{p}\left(\mathbf{C}^{d}\right)}, \quad w \in \mathbf{C}^{d} .
$$


Then $\left\|G_{K, C, \omega}\right\|_{L^{p, q}\left(\mathbf{C}^{2 d}\right)}=\left\|G_{K, p, \omega}\right\|_{L^{q}\left(\mathbf{C}^{d}\right)}$. Also let $K_{\omega}$ be as in $(3.2), F \in A_{E,\left(\omega_{1}\right)}^{p_{1}}\left(\mathbf{C}^{d}\right)$ and $H \in B_{E,\left(1 / \omega_{2}\right)}^{p_{2}}\left(\mathbf{C}^{d}\right)$, and set

$$
F_{\omega_{1}}(w) \equiv|F(w)| e^{-|w|^{2} / 2} \omega_{1}(\sqrt{2} \bar{w}), \quad w \in \mathbf{C}^{d}
$$

and

$$
H_{\omega_{2}}(z) \equiv|H(z)| e^{-|z|^{2} / 2} / \omega_{2}(\sqrt{2} \bar{z}), \quad z \in \mathbf{C}^{d} .
$$

By Hölder's inequality we get

$$
\begin{aligned}
\left|(T F, H)_{B^{2}}\right| & =\left|\int_{\mathbf{C}^{d}}(T F)(z) \overline{H(z)} e^{-|z|^{2}} d \lambda(z)\right| \\
& \leqslant \iint_{\mathbf{C}^{2 d}} K_{\omega}(z, w) F_{\omega_{1}}(w) H_{\omega_{2}}(z) d \lambda(z) d \lambda(w) \\
& =\iint_{\mathbf{C}^{2 d}} G_{K, C, \omega}(z, w) \Phi(z, w) d \lambda(z) d \lambda(w) \lesssim\left\|G_{K, C, \omega}\right\|_{L^{p, q}}\|\Phi\|_{L^{p^{\prime}, q^{\prime}}}
\end{aligned}
$$

where

$$
\begin{aligned}
& \Phi(x+i \xi, y+i \eta) \\
& =F_{\omega_{1}}\left(U_{d}^{-1}\left(C_{21}(x, \xi)+C_{22}(y, \eta)\right)\right) H_{\omega_{2}}\left(U_{d}^{-1}\left(C_{11}(x, \xi)+C_{12}(y, \eta)\right)\right), \quad x, y, \xi, \eta \in \mathbf{R}^{d} .
\end{aligned}
$$

Here we identify $(x, \xi) \in \mathbf{R}^{2 d}$ by corresponding $2 d \times 1$-matrix $\left(\begin{array}{l}x \\ \xi\end{array}\right)$, as usual.

We need to estimate $\|\Phi\|_{L^{p^{\prime}, q^{\prime}}}$, and start with reformulating $\|\Phi(\cdot, w)\|_{L^{p^{\prime}}}$. For $\|\Phi(\cdot, w)\|_{L^{p^{\prime}}}$ we take

$$
(x, \xi) \mapsto C_{21}\left((x, \xi)+C_{21}^{-1} C_{22}(y, \eta)\right)
$$

as new variables of integration, and get

$$
\|\Phi(\cdot, w)\|_{L^{p^{\prime}}}=\| F_{\omega_{1}} \cdot H_{\omega_{2}}\left(U_{d}^{-1}\left(B_{1}\left(\cdot-B_{2}(y, \eta)\right)\right) \|_{L^{p^{\prime}}}, \quad w=y+i \eta,\right.
$$

where $B_{1}$ and $B_{2}$ are the matrices

$$
B_{1}=C_{11} C_{21}^{-1} \in \mathbf{M}(2 d, \mathbf{R}) \quad \text { and } \quad B_{2}=C_{21} C_{11}^{-1} C_{12}-C_{22} \in \mathbf{M}(2 d, \mathbf{R}),
$$

which are invertible due to Remark 3.1 and the assumptions. Hence, for $F_{\omega_{1}}^{0}=$ $F_{\omega_{1}}\left(U_{d}^{-1} \cdot\right)$ and $H_{\omega_{2}}^{0}=H_{\omega_{2}} \circ U_{d}^{-1} \circ\left(-B_{1}\right)$ we have

$$
\|\Phi(\cdot, w)\|_{L^{p^{\prime}}}=\left(\left(\left|F_{\omega_{1}}^{0}\right|^{p^{\prime}} *\left|H_{\omega_{2}}^{0}\right|^{p^{\prime}}\right)\left(B_{2}(y, \eta)\right)\right)^{\frac{1}{p^{\prime}}}, \quad w=y+i \eta .
$$

If $\boldsymbol{r}_{1}=\boldsymbol{p}_{1} / p^{\prime}$ and $\boldsymbol{r}_{2}=\boldsymbol{p}_{2}^{\prime} / p^{\prime}$, then it follows from (3.4) that

$$
\frac{1}{\boldsymbol{r}_{1}}+\frac{1}{\boldsymbol{r}_{2}}=1+\frac{p^{\prime}}{q^{\prime}}, \quad \text { and } \quad \boldsymbol{r}_{1}, \boldsymbol{r}_{2}, \frac{q^{\prime}}{p^{\prime}} \geqslant 1
$$

Hence, by (3.10), $q^{\prime} / p^{\prime} \geqslant 1$, the fact that $B_{2}$ is invertible, and Hölder's and Young's inequalities we obtain

$$
\begin{aligned}
& \|\Phi\|_{L^{p^{\prime}, q^{\prime}}} \lesssim\left\|\left(\left(\left|F_{\omega_{1}}^{0}\right|^{p^{\prime}} *\left|H_{\omega_{2}}^{0}\right|^{p^{\prime}}\right)\left(B_{2} \cdot\right)\right)^{\frac{1}{p^{\prime}}}\right\|_{L^{q^{\prime}}}=\left(\left\|\left|F_{\omega_{1}}^{0}\right|^{p^{\prime}} *\left|H_{\omega_{2}}^{0}\right|^{p^{\prime}}\right\|_{L_{E}^{q^{\prime} / p^{\prime}}}\right)^{\frac{1}{p^{\prime}}} \\
& \leqslant\left(\left\|\left|F_{\omega_{1}}^{0}\right|^{p^{\prime}}\right\|_{L_{E}^{r_{1}} \|}\left\|\left.H_{\omega_{2}}^{0}\right|^{p^{\prime}}\right\|_{L_{E}^{r_{2}}}\right)^{\frac{1}{p^{\prime}}}=\left\|F_{\omega_{1}}\right\|_{L_{E}^{p_{1}}}\left\|H_{\omega_{2}}\right\|_{L_{E}^{p_{2}^{\prime}}}
\end{aligned}
$$

and the right-hand side of (3.8) follows by taking the supremum over all such $H$ with $\|H\|_{B_{E,\left(1 / \omega_{2}\right)}^{p_{2}^{\prime}}} \leqslant 1$. 
The existence of extension now follows from Hahn-Banach's theorem. By these estimates it also follows that

$$
(z, w) \mapsto K_{\omega}(z, w) F_{\omega_{1}}(w) \overline{H_{\omega_{2}}(z)}
$$

belongs to $L^{1}\left(\mathbf{C}^{d} \times \mathbf{C}^{d}\right)$, and the uniqueness is a straight-forward application of Lebesgue's theorem.

For corresponding pseudo-differential operator with symbol $a$, the kernel is given by $K(z, w)=e^{(z, w)} a(z, w)$. By straight-forward computations it follows that $G_{K, C, \omega, p}$ takes the form

$$
G_{K, C, \omega, p}(w)=\left\|a_{\omega}(\cdot, w)\right\|_{L^{p}\left(\mathbf{C}^{d}\right)}, \quad w \in \mathbf{C}^{d},
$$

when $C_{11}=C_{12}=C_{22}=I_{2 d}$ and $C_{21}=0$, or $C_{11}=C_{12}=C_{21}=I_{2 d}$ and $C_{22}=0$, where

$$
a_{\omega}(z, w)=e^{-\frac{1}{2} \cdot|z|^{2}} a(z+w, w) \omega(\sqrt{2}(\overline{z+w}), \sqrt{2} w)
$$

or

$$
a_{\omega}(z, w)=e^{-\frac{1}{2} \cdot|w|^{2}} a(z+w, z) \omega(\sqrt{2}(\overline{z+w}), \sqrt{2} z) .
$$

Hence, Theorem 3.3 gives the following.

Theorem 3.4. Let $\omega_{1}$ and $\omega_{2}$ be weights on $\mathbf{C}^{d}$, $\omega$ be a weight on $\mathbf{C}^{d} \times \mathbf{C}^{d}$ such that (3.3) holds, $\boldsymbol{p}_{1}, \boldsymbol{p}_{2}, p$ and $q$ be as in (3.4). Also let $a \in \widehat{A}\left(\mathbf{C}^{d} \times \mathbf{C}^{d}\right)$ and let $a_{\omega}$ be given by (3.11) or by (3.12) for $z, w \in \mathbf{C}^{d}$. If $a_{\omega} \in L^{p, q}\left(\mathbf{C}^{2 d}\right)$, then the operator $\operatorname{Op}_{\mathfrak{V}}(a)$ in (2.4) from $\mathcal{A}_{b_{1}}\left(\mathbf{C}^{d}\right)$ to $A\left(\mathbf{C}^{d}\right)$ is uniquely extendable to a continuous mapping from $A_{E,\left(\omega_{1}\right)}^{\boldsymbol{p}_{1}}\left(\mathbf{C}^{d}\right)$ to $A_{E,\left(\omega_{2}\right)}^{\boldsymbol{p}_{2}}\left(\mathbf{C}^{d}\right)$.

We also have the following result related to Theorem 3.3. Here the matrix $C$ is given by (3.1) with

$$
C_{11}=C_{21}=I_{2 d}, \quad C_{12}=\left(\begin{array}{cc}
0 & 0 \\
0 & I_{d}
\end{array}\right) \quad \text { and } \quad C_{22}=\left(\begin{array}{cc}
I_{d} & 0 \\
0 & 0
\end{array}\right)
$$

which obviously satisfies (3.5). Also again recall Remark 1.16 for notations.

Theorem 3.5. Let $C$ be given by (3.1) with $C_{j k}$ given by (3.13), $\omega_{1}$ and $\omega_{2}$ be weights on $\mathbf{C}^{d}, \omega$ be a weight on $\mathbf{C}^{d} \times \mathbf{C}^{d}$ such that (3.3) holds, and let $p, q \in[1, \infty]$. Also let $K \in \widehat{A}\left(\mathbf{C}^{d} \times \mathbf{C}^{d}\right)$ and $G_{K, C, \omega}$ be as in (3.2). If $G_{K, C, \omega} \in L_{*}^{p, q}\left(\mathbf{C}^{2 d}\right)$, then $T_{K}$ in (2.1) from $\mathcal{A}_{b_{1}}\left(\mathbf{C}^{d}\right)$ to $A\left(\mathbf{C}^{d}\right)$ is uniquely extendable to a continuous mapping from $A_{\left(\omega_{1}\right)}^{p^{\prime}, q^{\prime}}\left(\mathbf{C}^{d}\right)$ to $A_{*,\left(\omega_{2}\right)}^{q, p}\left(\mathbf{C}^{d}\right)$, and

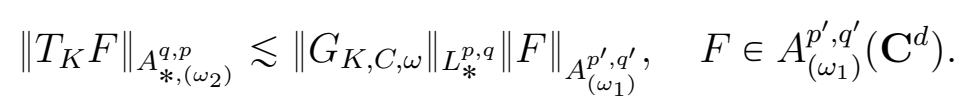

Proof. Let $F_{\omega_{1}}, F_{\omega_{1}}^{0}, H_{\omega_{2}}$ and $H_{\omega_{2}}^{0}$ be the same as in the proof of Theorem 3.3, and let $K_{\omega}$ be as in (3.2). Then

$$
\begin{aligned}
\left|\left(T_{K} F, H\right)_{B^{2}}\right| & \leqslant \iiint \int K_{\omega}(x, \xi, y, \eta) F_{\omega_{1}}^{0}(y, \eta) H_{\omega_{2}}^{0}(x, \xi) d x d \xi d y d \eta \\
& \left.=\iiint \int G_{K, C, \omega}(x, \xi, y, \eta)\right) F_{\omega_{1}}^{0}(x+y, \xi) H_{\omega_{2}}^{0}(x, \xi+\eta) d x d \xi d y d \eta \\
& \leqslant\left\|G_{K, C, \omega}\right\|_{L_{*}^{p, q}}\left\|\Phi_{0}\right\|_{L^{p^{\prime}}}
\end{aligned}
$$


where

$$
\begin{aligned}
\Phi_{0}(x, \xi) & =\left(\iint\left|F_{\omega_{1}}^{0}(x+y, \xi) H_{\omega_{2}}^{0}(x, \xi+\eta)\right|^{q^{\prime}} d y d \eta\right)^{\frac{1}{q^{\prime}}} \\
& =\left\|F_{\omega_{1}}^{0}(\cdot, \xi)\right\|_{L^{q^{\prime}}}\left\|H_{\omega_{2}}^{0}(x, \cdot)\right\|_{L^{q^{\prime}}}, \quad x, \xi \in \mathbf{R}^{d} .
\end{aligned}
$$

Hence,

$$
\left\|\Phi_{0}\right\|_{L^{p^{\prime}}}=\left\|F_{\omega_{1}}\right\|_{L^{q^{\prime}, p^{\prime}}}\left\|H_{\omega_{2}}\right\|_{L_{*}^{p^{\prime}, q^{\prime}}} .
$$

The continuity assertion now follows from these estimates, an application of HahnBanach's theorem and Lebesgue's theorem (cf. the end of the proof of Theorem 3.3).

Remark 3.6. In Theorem 3.5, the matrix $C$ is chosen only as (3.1) and (3.13), while Theorem 3.3 is valid for a whole family of matrices with the only restriction (3.5) or (3.6). On the other hand, by similar arguments, it follows that the conclusions in Theorem 3.5 are still true when, more generally, $C \in \mathbf{M}(4 d, \mathbf{R})$ is of the form

$$
\begin{aligned}
& \left(\begin{array}{cccc} 
\pm I_{d} & 0 & \pm I_{d} & 0 \\
0 & \pm I_{d} & 0 & 0 \\
\pm I_{d} & 0 & 0 & 0 \\
0 & \pm I_{d} & 0 & \pm I_{d}
\end{array}\right), \\
& \left(\begin{array}{cccc} 
\pm I_{d} & 0 & \pm I_{d} & 0 \\
0 & 0 & 0 & \pm I_{d} \\
0 & 0 & \pm I_{d} & 0 \\
0 & \pm I_{d} & 0 & \pm I_{d}
\end{array}\right) \quad \text { or } \quad\left(\begin{array}{cccc} 
\pm I_{d} & 0 & 0 & 0 \\
0 & \pm I_{d} & 0 & \pm I_{d} \\
\pm I_{d} & 0 & \pm I_{d} & 0 \\
0 & \pm I_{d} & 0 & 0
\end{array}\right)
\end{aligned}
$$

for any choice of \pm at each place, provided the mixed Lebesgue conditions on $G_{K, C, \omega}$ are slightly modified.

In order to apply Theorem 3.3 to real pseudo-differential operators we have the following.

Lemma 3.7. Let $\phi(x, \xi)=\pi^{-\frac{d}{2}} e^{i\langle x, \xi\rangle} e^{-\frac{1}{2}\left(|x|^{2}+|\xi|^{2}\right)}, x, \xi \in \mathbf{R}^{d}, a \in \mathcal{H}_{b_{1}}^{\prime}\left(\mathbf{R}^{2 d}\right)$ and let $K_{a}$ be the kernel of $\operatorname{Op}(a)$. Then

$$
\begin{aligned}
& e^{-\frac{1}{2}\left(|z|^{2}+|w|^{2}\right)} \mathfrak{V}_{\Theta, d} K_{a}(z, w) \\
& =(2 \pi)^{\frac{d}{2}} e^{-i(\langle x, \xi-2 \eta\rangle+\langle y, \eta\rangle)}\left(V_{\phi} a\right)(\sqrt{2} x,-\sqrt{2} \eta, \sqrt{2}(\eta-\xi), \sqrt{2}(y-x))
\end{aligned}
$$

when $z=x+i \xi \in \mathbf{C}^{d}$ and $w=y+i \eta \in \mathbf{C}^{d}$.

Proof. Let $\phi_{0}(x, \xi)=\pi^{-\frac{d}{2}} e^{-\frac{1}{2}\left(|x|^{2}+|\xi|^{2}\right)}=e^{-i\langle x, \xi\rangle} \phi(x, \xi)$. By formal computations and Fourier's inversion formula we get

$$
\begin{aligned}
& (2 \pi)^{d} e^{\frac{i}{2} \cdot(\langle x, \xi\rangle-\langle y, \eta\rangle)} e^{-\frac{1}{4}\left(|z|^{2}+|w|^{2}\right)} \mathfrak{V}_{\Theta, d} K_{a}(z / \sqrt{2}, w / \sqrt{2}) \\
& =\iiint a\left(x_{1}, \xi_{1}\right) e^{i\left\langle x_{1}-y_{1}, \xi_{1}\right\rangle} \phi_{0}\left(x_{1}-x, y_{1}-y\right) e^{-i\left(\left\langle y_{1}, \eta\right\rangle-\left\langle x_{1}, \xi\right\rangle\right)} d x_{1} d y_{1} d \xi_{1} \\
& =(2 \pi)^{\frac{d}{2}} e^{-i\langle y, \eta\rangle} \iint a\left(x_{1}, \xi_{1}\right) \phi_{0}\left(x_{1}-x, \xi_{1}+\eta\right) e^{i\left\langle x_{1}, \xi_{1}\right\rangle} e^{-i\left(\left\langle y, \xi_{1}\right\rangle-\left\langle x_{1}, \xi\right\rangle\right)} d x_{1} d \xi_{1} \\
& =(2 \pi)^{\frac{d}{2}} e^{i\langle x-y, \eta\rangle} \iint a\left(x_{1}, \xi_{1}\right) \phi\left(x_{1}-x, \xi_{1}+\eta\right) e^{-i\left(\left\langle x_{1}, \eta-\xi\right\rangle+\left\langle y-x, \xi_{1}\right\rangle\right)} d x_{1} d \xi_{1} \\
& =(2 \pi)^{\frac{3 d}{2}} e^{i\langle x-y, \eta\rangle}\left(V_{\phi} a\right)(x,-\eta, \eta-\xi, y-x) .
\end{aligned}
$$


We can now use the previous lemma and theorems to obtain mapping properties for pseudo-differential operators with symbols in modulation spaces. For example, we may combine Lemma 3.7 and Theorem 3.3 to deduce the following result, which is the same as [28, Theorem 2.2]. Hence our kernel results on the Bargmann transform side can be used to regain classical mapping properties pseudo-differential operators when acting on modulation spaces.

Theorem 3.8. Let $E$ be an ordered basis of $\mathbf{R}^{2 d}, A \in \mathbf{M}(\mathbf{R}, d), p, q \in[1, \infty]$ and $\boldsymbol{p}_{1}, \boldsymbol{p}_{2} \in[1, \infty]^{2 d}$ be as in $(3.4), \omega_{0} \in \mathscr{P}_{E}\left(\mathbf{R}^{4 d}\right)$ and $\omega_{1}, \omega_{2} \in \mathscr{P}_{E}\left(\mathbf{R}^{2 d}\right)$ be such that

$$
\frac{\omega_{2}\left(x-A y, \xi+\left(I-A^{*}\right) \eta\right)}{\omega_{1}\left(x+(I-A) y, \xi-A^{*} \eta\right)} \lesssim \omega_{0}(x, \xi, \eta, y), \quad x, y, \xi, \eta \in \mathbf{R}^{d},
$$

and let $a \in M_{\left(\omega_{0}\right)}^{p, q}\left(\mathbf{R}^{2 d}\right)$. Then $\mathrm{Op}_{A}(a)$ from $\Sigma_{1}\left(\mathbf{R}^{d}\right)$ to $\Sigma_{1}^{\prime}\left(\mathbf{R}^{d}\right)$ extends uniquely to continuous operator from $M_{E,\left(\omega_{1}\right)}^{\boldsymbol{p}_{1}}\left(\mathbf{R}^{d}\right)$ to $M_{E,\left(\omega_{2}\right)}^{\boldsymbol{p}_{2}}\left(\mathbf{R}^{d}\right)$, and

$$
\left\|\mathrm{Op}_{A}(a)\right\|_{M_{E,\left(\omega_{1}\right)}^{p_{1}} \rightarrow M_{E,\left(\omega_{2}\right)}^{p_{2}}} \lesssim\|a\|_{M_{\left(\omega_{0}\right)}^{p, q}} .
$$

Proof. By (1.40) and Proposition 1.19 we may assume that $A=0$. Let

$$
C_{11}=C_{21}=C_{22}=I_{2 d}, \quad C_{12}=0
$$

and let $\omega$ be given by

$$
\omega(x, \xi, y, \eta)=\omega_{0}(x,-\eta, \xi+\eta, y-x), \quad x, y, \xi, \eta \in \mathbf{R}^{d},
$$

which we identify with

$$
\omega(z, w), \quad z=x+i \xi \in \mathbf{C}^{d}, w=y+i \eta \in \mathbf{C}^{d} .
$$

Then it follows by straight-forward computations that (3.15) is the same as (3.3). Furthermore, let $K_{0}=\mathfrak{V}_{\Theta, d} K_{a}$, where $K_{a}$ is the kernel of the operator $\operatorname{Op}(a)$. Then it follows from Lemma 3.7 and straight-forward computations that if

$$
H_{a, \omega_{0}}(x, \xi, \eta, y)=\left|V_{\phi} a(x, \xi, \eta, y)\right| \cdot \omega_{0}(x, \xi, \eta, y), \quad x, y, \xi, \eta \in \mathbf{R}^{d}
$$

then

$$
\begin{aligned}
& H_{a, \omega_{0}}(\sqrt{2} x,-\sqrt{2}(\xi+\eta), \sqrt{2} \eta, \sqrt{2} y)=G_{K_{0}, C, \omega}(z, w), \\
& z=x+i \xi \in \mathbf{C}^{d}, w=y+i \eta \in \mathbf{C}^{d} .
\end{aligned}
$$

By first applying the $L^{p}$-norm on (3.16) with respect to $x$ and $\xi$, and thereafter applying the $L^{q}$-norm with respect to $y$ and $\eta$, we get

$$
\left\|G_{K_{0}, C, \omega}\right\|_{L^{p, q}\left(\mathbf{C}^{2 d}\right)}=\left\|H_{a, \omega_{0}}\right\|_{L^{p, q}\left(\mathbf{R}^{4 d}\right)}=\|a\|_{M_{\left(\omega_{0}\right)}^{p, q}}<\infty .
$$

Hence, the assumptions in Theorem 3.3 are fullfiled, and we conclude that the operator $T_{K_{0}}$ with kernel $K_{0}$ is continuous from $A_{E,\left(\omega_{1}\right)}^{p_{1}}\left(\mathbf{C}^{d}\right)$ to $A_{E,\left(\omega_{2}\right)}^{p_{2}}\left(\mathbf{C}^{d}\right)$. The asserted continuity for $\operatorname{Op}(a)$ is now a consequence of the commutative diagram

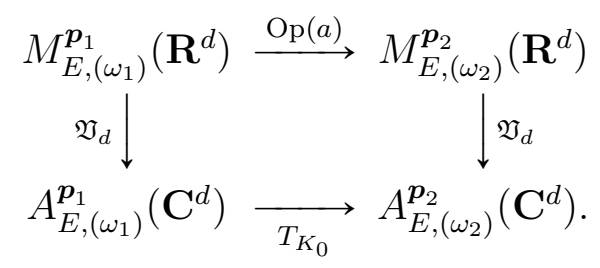

The next result extends [24, Theorem 3.3] and follows by similar arguments as in the previous proof, using Theorem 3.5 instead of Theorem 3.3. The details are left for the reader. 
Theorem 3.9. Let $\omega_{1}$ and $\omega_{2} \in \mathscr{P}_{E}\left(\mathbf{R}^{2 d}\right), \omega \in \mathscr{P}_{E}\left(\mathbf{R}^{4 d}\right)$ be such that

$$
\frac{\omega_{2}(x, \xi+\eta)}{\omega_{1}(x+y, \xi)} \lesssim \omega_{0}(x, \xi, \eta, y), \quad x, y, \xi, \eta \in \mathbf{R}^{d}
$$

let $p, q \in[1, \infty]$, and let $a \in W_{\left(\omega_{0}\right)}^{p, q}\left(\mathbf{R}^{2 d}\right)$. Then $\mathrm{Op}_{0}(a)$ from $\Sigma_{1}\left(\mathbf{R}^{d}\right)$ to $\Sigma_{1}^{\prime}\left(\mathbf{R}^{d}\right)$ is uniquely extendable to a continuous mapping from $\left.M_{\left(\omega_{1}\right)}^{q^{\prime}, p^{\prime}}\left(\mathbf{R}^{d}\right)\right)$ to $\left.W_{\left(\omega_{2}\right)}^{p, q}\left(\mathbf{R}^{d}\right)\right)$, and

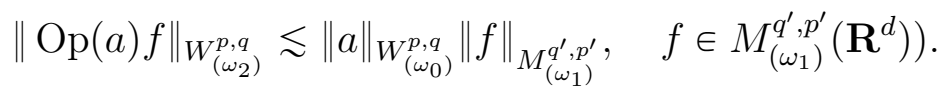

Remark 3.10. Let $E, p, q, \boldsymbol{p}_{j}, \phi_{0}$ and $\phi$ be the same as in Lemma 3.7, Theorem 3.8 and their proofs. Also let $A \in \mathbf{M}(\mathbf{R}, d)$ and let $\phi_{A}=e^{i\left\langle A D_{\xi}, D_{x}\right\rangle} \phi$. Then the condition on $a$ in Theorem 3.8 is that $\left\|V_{\phi_{0}} a \cdot \omega_{0}\right\|_{L^{p, q}}<\infty$. In view of $[5,6,29]$ and Proposition 1.13 (2), the previous condition is the same as $\left\|V_{\phi_{A}} a \cdot \omega_{0}\right\|_{L^{p, q}}<\infty$ because $\omega_{0}$ is moderate.

We observe that all weights in Theorem 3.8 are moderate, while there are no such assumptions or other restrictions on the involved weight functions in Theorem 3.3. Since the latter result is used to prove the former one, a natural question is wether Theorem 3.8 can be extended to broader classes of weight functions. In view of Remark 1.14, it is evident that the imposing moderate conditions on weights might in some context be considered as strong restrictions.

The answer on this question is affirmative in the sense that for suitable modifications, the moderate conditions on the weights in Theorem 3.8 can be removed.

In fact, let $\mathcal{H}_{b_{1}}^{A}\left(\mathbf{R}^{2 d}\right)$ be the modification of $\mathcal{H}_{b_{1}}\left(\mathbf{R}^{2 d}\right)$, given by

$$
\mathcal{H}_{b_{1}}^{A}\left(\mathbf{R}^{2 d}\right)=\left\{e^{i\left\langle A D_{\xi}, D_{x}\right\rangle}\left(e^{i\langle x, \xi\rangle} a\right) ; a \in \mathcal{H}_{b_{1}}\left(\mathbf{R}^{2 d}\right)\right\},
$$

$\left(\mathcal{H}_{b_{1}}^{A}\right)^{\prime}\left(\mathbf{R}^{2 d}\right)$ be the dual of $\mathcal{H}_{b_{1}}^{A}\left(\mathbf{R}^{2 d}\right), \omega_{1}, \omega_{2}$ be weights on $\mathbf{R}^{2 d}$ and let $\omega_{0}$ be a weight on $\mathbf{R}^{4 d}$ such that (3.15) holds. Then it follows from the proof of Theorem 3.8 that the following is true:

- if $a \in\left(\mathcal{H}_{b_{1}}^{A}\right)^{\prime}\left(\mathbf{R}^{2 d}\right)$, then $V_{\phi_{A}} a$ makes sense as a smooth function;

- if $a \in\left(\mathcal{H}_{b_{1}}^{A}\right)^{\prime}\left(\mathbf{R}^{2 d}\right)$ satisfies $\left\|V_{\phi_{A}} a \cdot \omega_{0}\right\|_{L^{p, q}}<\infty$, then $\mathrm{Op}_{A}(a)$ from $\mathcal{H}_{b_{1}}\left(\mathbf{R}^{d}\right)$ to $\mathcal{H}_{b_{1}}^{\prime}\left(\mathbf{R}^{d}\right)$ extends uniquely to a continuous operator from $M_{E,\left(\omega_{1}\right)}^{p_{1}}\left(\mathbf{R}^{d}\right)$ to $M_{E,\left(\omega_{2}\right)}^{p_{2}}\left(\mathbf{R}^{d}\right)$.

In similar ways, Theorem 3.9 can be extended to permit more general weight classes.

\section{References}

[1] Bargmann, V.: On a Hilbert space of analytic functions and an associated integral transform. - Comm. Pure Appl. Math. 14, 1961, 187-214.

[2] Bargmann, V.: On a Hilbert space of analytic functions and an associated integral transform. Part II. A family of related function spaces. Application to distribution theory. - Comm. Pure Appl. Math. 20, 1967, 1-101.

[3] Bauer, W.: Berezin-Toeplitz quantization and composition formulas. - J. Funct. Anal. 256, 2007, 3107-3142.

[4] Berezin, F. A.: Wick and anti-Wick symbols of operators. - Mat. Sb. (N.S.) 86, 1971, 578-610.

[5] Cappiello, M., and J. TofT: Pseudo-differential operators in a Gelfand-Shilov setting. Math. Nachr. 290, 2017, 738-755. 
[6] Carypis, E., and P. Wahlberg: Propagation of exponential phase space singularities for Schrödinger equations with quadratic Hamiltonians. - J. Fourier Anal. Appl. 23, 2017, 530571.

[7] Chen, Y., M. Signahl, and J. TofT: Factorizations and singular value estimates of operators with Gelfand-Shilov and Pilipović kernels. - J. Fourier Anal. Appl. 24, 2018, 666-698.

[8] Cordero, E., S. Pilipović, L. Rodino, and N. Teofanov: Quasianalytic Gelfand-Shilov spaces with applications to localization operators. - Rocky Mountain J. Math. 40, 2010, 11231147.

[9] Feichtinger, H. G.: Banach spaces of distributions of Wiener's type and interpolation. In: Proc. Conf. Oberwolfach, Functional Analysis and Approximation, August 1980 (edited by P. Butzer, B. Sz. Nagy and E. Görlich), Int. Ser. Num. Math. 69, Birkhäuser Verlag, Basel, Boston, Stuttgart, 1981, 153-165.

[10] Feichtinger, H. G.: Modulation spaces on locally compact abelian groups. - Technical report, University of Vienna, Vienna, 1983; also in: Wavelets and their applications (edited by M. Krishna, R. Radha and S. Thangavelu), Allied Publishers Private Limited, NewDehli Mumbai Kolkata Chennai Hagpur Ahmedabad Bangalore Hyderbad Lucknow, 2003, 99-140.

[11] Fernandez, C., A. GalBis, and J. TofT: The Bargmann transform and powers of harmonic oscillator on Gelfand-Shilov subspaces. - Rev. R. Acad. Cienc. Exactas Fís. Nat. Ser. A Mat. RACSAM 111, 2017, 1-13.

[12] Gröchenig, K.: Foundations of time-frequency analysis. - Birkhäuser, Boston, 2001.

[13] Gröchenig, K.: Weight functions in time-frequency analysis - In: Pseudodifferential operators: Partial differential equations and time-frequency analysis (edited by L. Rodino and M. W. Wong), Fields Inst. Commun. 52, 2007, 343-366.

[14] Gröchenig, K., and G. Zimmermann: Spaces of test functions via the STFT. - J. Funct. Spaces Appl. 2, 2004, 25-53.

[15] Hörmander, L.: The analysis of linear partial differential operators, vol. I-III. - SpringerVerlag, Berlin Heidelberg NewYork Tokyo, 1983, 1985.

[16] Lozanov Crvenković, Z., and D. Perišić: Hermite expansions of elements of Gelfand Shilov spaces in quasianalytic and non quasianalytic case. - Novi Sad J. Math. 37, 2007, 129-147.

[17] Pilipović, S.: Generalization of Zemanian spaces of generalized functions which have orthonormal series expansions. - SIAM J. Math. Anal. 17, 1986, 477-484.

[18] Pilipović, S.: Tempered ultradistributions. Boll. Unione Mat. Ital. 7, 1988, 235-251.

[19] Reed, M., and B. Simon: Methods of modern mathematical physics. - Academic Press, London New York, 1979.

[20] Ruzhansky, M., and N. Tokmagambetov: Nonharmonic analysis of boundary value problems. - Int. Math. Res. Notices 12, 2016, 3548-3615.

[21] Teofanov, N.: Ultradistributions and time-frequency analysis. - In: Pseudo-differential operators and related topics (edited by P. Boggiatto, L. Rodino, J. Toft and M. W. Wong), Oper. Theory Adv. Appl. 164, Birkhäuser, Basel, 2006, 173-192.

[22] Teofanov, N.: Gelfand-Shilov spaces and localization operators. - Funct. Anal. Approx. Comput. 7, 2015, 135-158.

[23] Tofт, J.: Continuity properties for modulation spaces with applications to pseudo-differential calculus, II. - Ann. Global Anal. Geom. 26, 2004, 73-106.

[24] Toft, J.: Pseudo-differential operators with symbols in modulation spaces. - In: PseudoDifferential operators: Complex analysis and partial differential equations (edited by B.-W. Schulze and M.W. Wong), Oper. Theory Adv. Appl. 205, Birkhäuser Verlag, Basel, 2010, $223-234$.

[25] TofT, J.: The Bargmann transform on modulation and Gelfand-Shilov spaces, with applications to Toeplitz and pseudo-differential operators. - J. Pseudo-Differ. Oper. Appl. 3, 2012, $145-227$. 
[26] Toft, J.: Images of function and distribution spaces under the Bargmann transform. - J. Pseudo-Differ. Oper. Appl. 8, 2017, 83-139.

[27] Tofт, J.: Continuity and compactness for pseudo-differential operators with symbols in quasiBanach spaces or Hörmander classes. - Anal. Appl. 15, 2017, 353-389.

[28] Toft, J.: Matrix parameterized pseudo-differential calculi on modulation spaces - In: Generalized functions and Fourier analysis (edited by M. Oberguggenberger, J. Toft, J. Vindas and P. Wahlberg), Oper. Theory Adv. Appl. 260, Birkhäuser, Basel Heidelberg New York Dordrecht London, 215-235.

[29] Tranquilli, G.: Global normal forms and global properties in function spaces for second order Shubin type operators. - PhD Thesis, 2013.

Received 12 February 2019 • Accepted 22 March 2019 